\title{
Big Data, Machine Learning, Artificial Intelligence
}

\author{
Various , Lexie J Byrd
}

May 2020

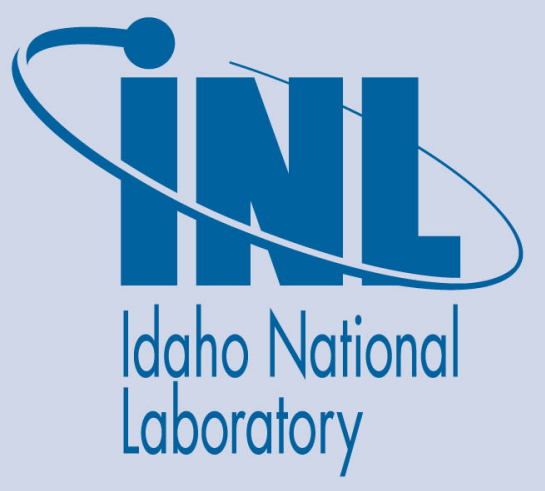

The INL is a U.S. Department of Energy National Laboratory operated by Battelle Energy Alliance 


\title{
Big Data, Machine Learning, Artificial Intelligence
}

\author{
Various , Lexie J Byrd
}

May 2020

Idaho National Laboratory Idaho Falls, Idaho 83415

http://www.inl.gov

Prepared for the U.S. Department of Energy Office of Nuclear Energy Under DOE Idaho Operations Office Contract DE-AC07-05ID14517 


\section{Big Data, Machine Learning, Artificial Intelligence}

NS\&T ML-AI

\section{INL - ML \& Al Symposium}

\section{April 17, 2020}

Purpose of Meeting:

- Introduce the topic of ML and AI to INL researchers

- Provide examples of how ML an Al are being applied across other industries

- Discuss current ML \& Al research and capabilities at INL

- Discuss planned activities, including engagement opportunities and collaboration opportunities

Presentations will include:

- Provide overview on Topic Area;

- Describe the status of industry

- Identify Issues (if any) and potential impact

- High level discussion of planned activities and outcomes 


\section{Big Data, Machine Learning, Artificial Intelligence}

\section{NS\&T ML-AI}

\section{Agenda for Machine Learning and Artificial Intelligence Symposium}

Friday, April 17th, 2020;

\begin{tabular}{|l|l|l|}
\hline \multicolumn{1}{|c}{ Time } & Welcome, Introductions, and Agenda & Curtis Smith \\
\hline $11: 00$ & What is Al? & R. Kunz \\
\hline $11: 25$ & Al, ML, and Statistics, oh My! & N. Lybeck \\
\hline $11: 35$ & Modeling Human Cognition: It's Not All Machine Learning & R. Boring \\
\hline $11: 45$ & Smart Reactors & Humberto Garcia \\
\hline $11: 55$ & Al in Robotics and Applying Natural Connections & V. Walker \\
\hline $12: 05$ & Al as Automation & K. Le Blanc \\
\hline $12: 15$ & ML in current projects & V. Agarwal \\
\hline $12: 25$ & ML in current projects & A. Al Rashdan \\
\hline $12: 35$ & $\begin{array}{l}\text { HPC Building a Scientific Language Model - Leveraging } \\
\text { ArXive.org research data and RoBERTa }\end{array}$ & C. Krome \\
\hline $12: 45$ & $\begin{array}{l}\text { Reverse engineering of stripped binaries using scalable deep } \\
\text { learning }\end{array}$ & M. Anderson \\
\hline
\end{tabular}




\section{Curtis Smith}

Group: Division Director for Nuclear Safety and Regulatory Research

Education: BS, MS, and PhD in Nuclear Engineering at ISU and MIT

\section{Presentation Overview}

Motivation for Al/ML in science, math, and engineering

- How AI/ML has advanced in the science, math, and engineering communities and how these advances may be used with INL applications such as computational risk assessment.

- These topics provide an insight into the potential for advanced analysis and operations for complex systems.

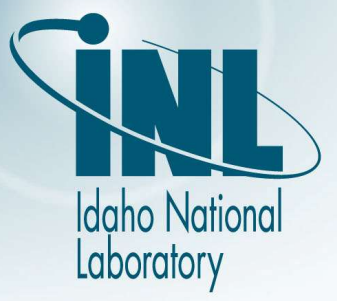




\section{My Motivation for Al/ML in Science, Math, and Engineering}

Dr. Curtis Smith, Director Nuclear Safety and Regulatory Research Division Idaho National Laboratory

A discussion on:

How Al/ML has advanced in science, math, \& engineering

How these advances may be used with INL applications such as computational risk assessment

The potential for advanced analysis and operations for complex systems 


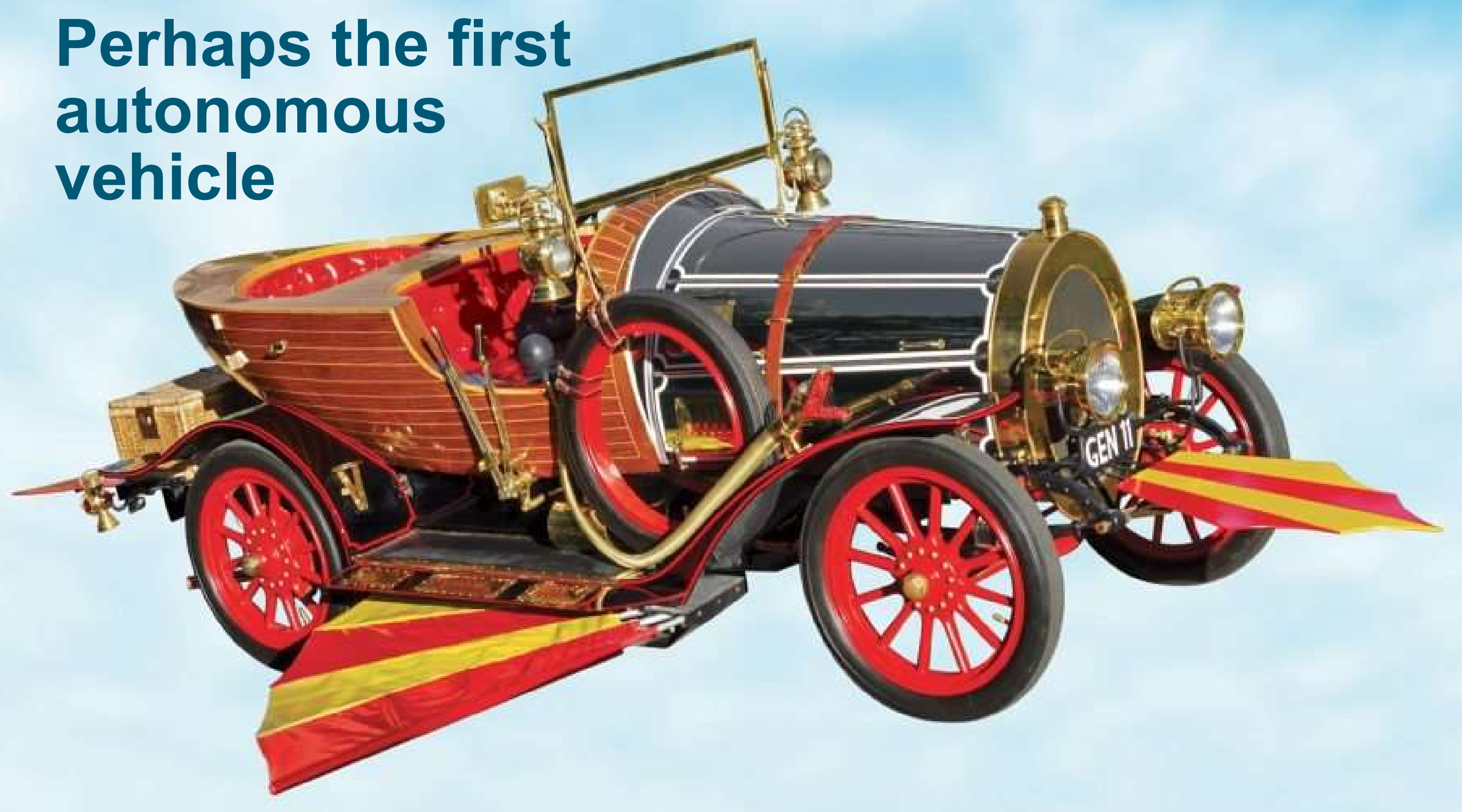




\section{What is Machine Learning/Artificial Intelligence (ML/AI)?}

- From Source of All Knowledge ${ }^{\mathrm{TM}} \rightarrow$ Wikipedia

- Artificial intelligence (Al) is intelligence demonstrated by machines

- Study of "intelligent agents": device that perceives its environment and takes actions that maximize its chance of successfully achieving its goals

- Machines that mimic "cognitive" functions that humans associate with the human mind, such as "learning" and "problem solving"

- Machine learning (ML) is the scientific study of algorithms and statistical models to perform a specific task without using explicit instructions, relying on patterns and inference instead

- Subset of artificial intelligence

- Builds a mathematical model based on sample data ("training data") to make predictions or decisions without being explicitly programmed to perform the task

- Closely related to computational statistics, which focuses on making predictions using computers 
A question $\rightarrow$ can we use Al/ML for Science, Math, and Engineering??
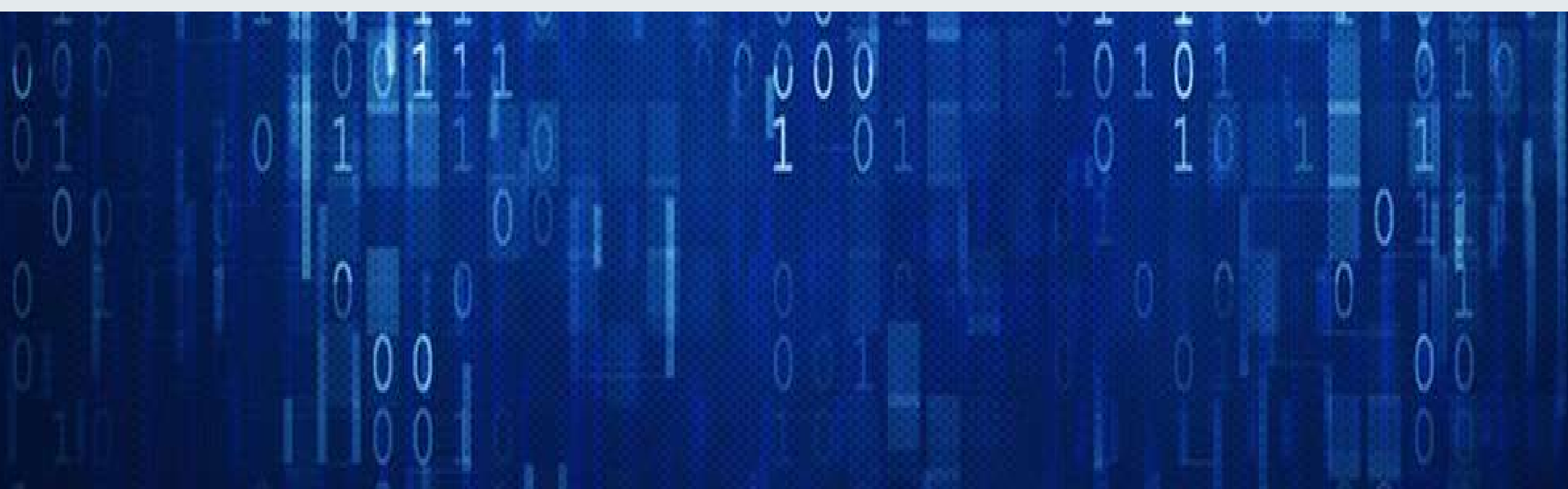


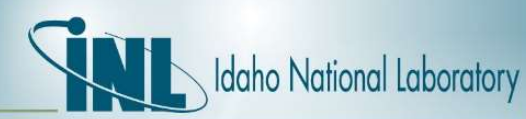

\section{Examples of current ML and Al applications}

- Symbolic reasoning to differentiate \& integrate math

- Neural network used 80 million examples of $1^{\text {st }}$ - and $2^{\text {nd }}$-order differential equations $\& 20$ million examples of integrated by parts

- How well does it work?

$$
y^{\prime}=\frac{16 x^{3}-42 x^{2}+2 x}{\left(-16 x^{8}+112 x^{7}-204 x^{6}+28 x^{5}-x^{4}+1\right)^{1 / 2}}
$$

- Significantly outperforms Mathematica (on integration, close to $100 \%$ accuracy)

- Mathematica reaches $85 \%$, Maple and Matlab perform less well

- In many cases, conventional solvers unable to find a solution in 30 seconds

- The neural net takes about a second to find its solutions

- https://www.technologyreview.com/s/614929/facebook-has-a-neural-network-that-can-do-advanced-math/

\section{- AlphaGo and AlphaGo Zero to play Go}

- AlphaGo defeated 18-time world champion Lee Sedol 4 games to 1

- Used game tree search, neural network trained on expert human games, second neural network for board positions, and additional Monte Carlo rules

- AlphaGo Zero used same tree search algorithm, but then single neural network trained without any human games

AlphaGo Zero defeated AlphaGo 100 games to 0

https://medium.com/ww-engineering/alphago-zero-a-brief-summary-dcff16ba3064 


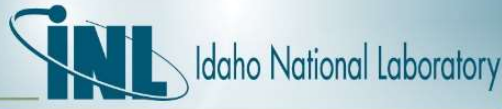

\section{How can these approaches help future risk-informed applications?}

- Recent nuclear power challenges have been mostly on economics and safety

- Need to provide new cost-beneficial approaches to safety via modern methods/tools/data

- We want to attract the next generation of scientists/engineers via these new approaches

- Computational Risk Assessment (CRA) is a combination of

- Probabilistic (i.e., dynamic) scenarios where they unfold and are not defined a priori

- Mechanistic analysis representing physics of the unfolding scenarios

- Idea $\rightarrow$ CRA to produce "synthetic data" for ML

- ML requires training data - however risk \& reliability have a small set of "failure" data

- CRA can explore rich space of normal \& off-normal conditions

- CRA can produce very large sets of synthetic data

- Idea $\rightarrow$ Digital regulator

- Agent-based systems for oversight of operations

- CRA + real-world sensors $\rightarrow$ next-gen regulation

- Keep an independent, digital presence in systems

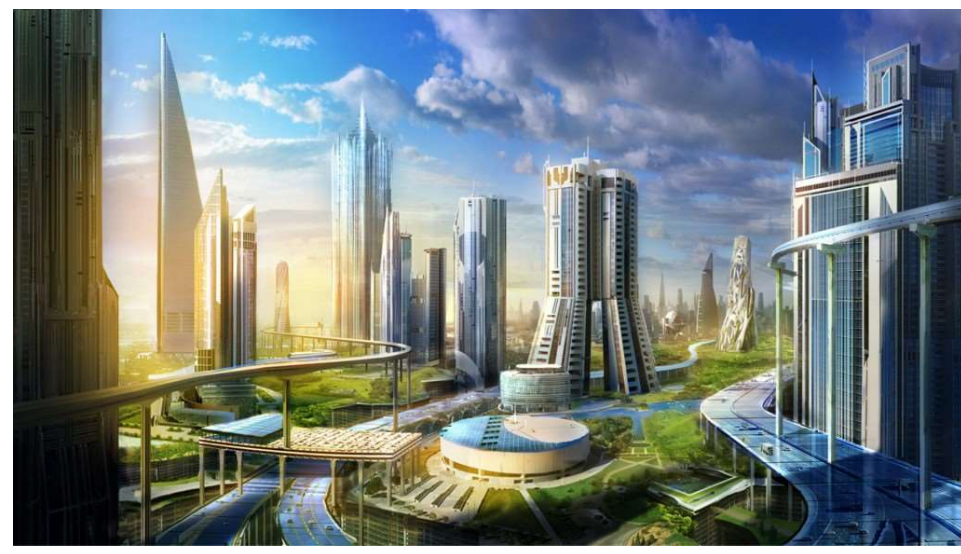




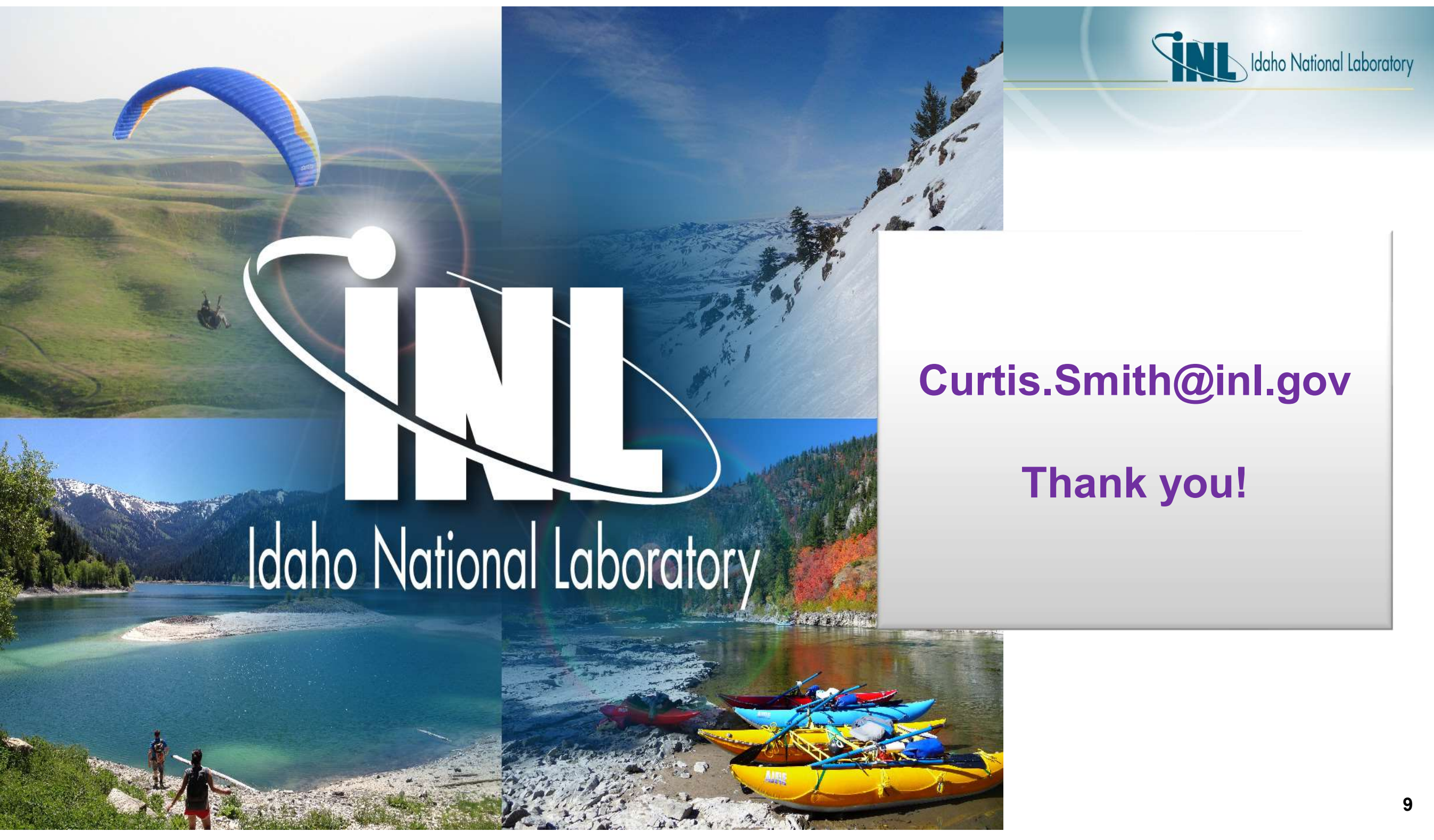




\section{Ross Kunz}

Group: Advanced Analytics

Education: PhD Statistics

Work focused in: Machine learning for chemistry and physics

(catalysts, batteries, materials)

Presentation Overview

What is Al?

- Overview of Al and the connection to Modeling/Simulation

- Understanding of complex data sets and discovery of new information

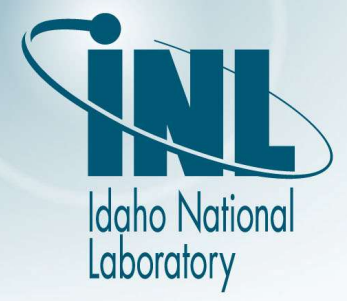




\section{Machine Learning \& Artificial Intelligence Symposium April 17, 2020}

Ross Kunz

B652 Advanced Analytics What is Al? 


\section{Definition}

- The capability of a machine to imitate intelligent human behavior

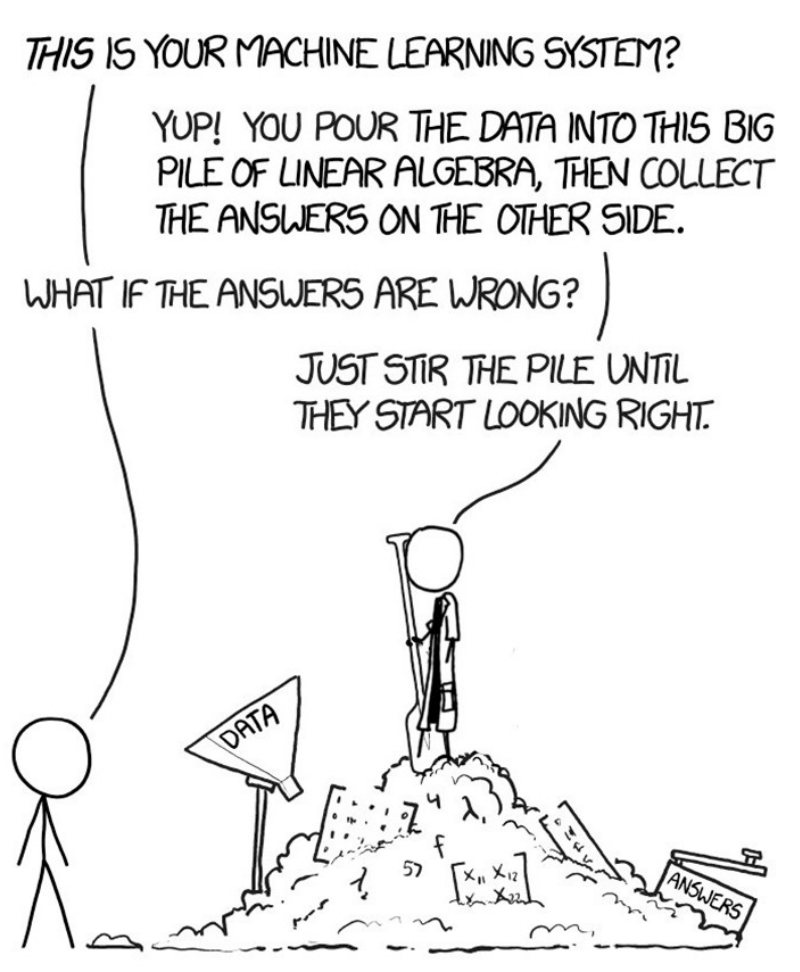

1. Data (kind of a big deal)

1. Good

2. Bad

3. Ugly

2. Domain problem

1. Data Structures

2. What information can be leveraged

3. No free lunch!

3. Results

1. I don't care, predict the cat!

2. The journey, not the destination that matters

Source: xkcd.com 


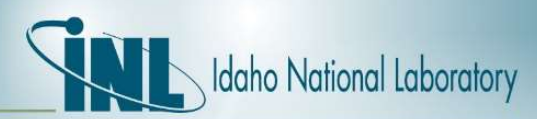

\section{Connection to Science}

\section{Data Analysis Spectrum}

- Little to No Data

- Strong Assumptions

- Highly Informative

- High Computation

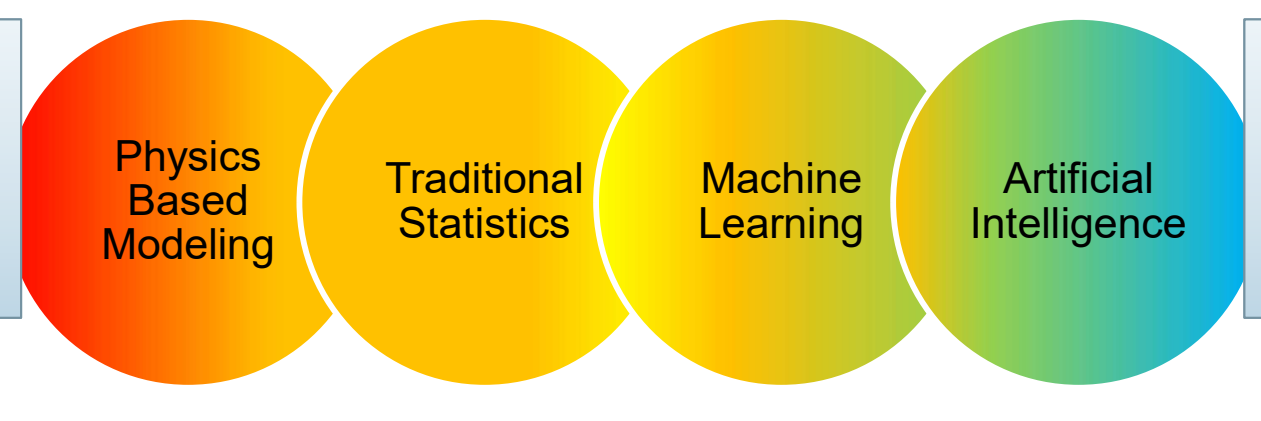

- Extreme Amounts of Data

- Little to No Assumptions

- Highly Predictive

- High Computation

\section{Physics to physics}

\section{Surrogate modeling}

\section{Experimental Discovery}




\section{Types of Problems}

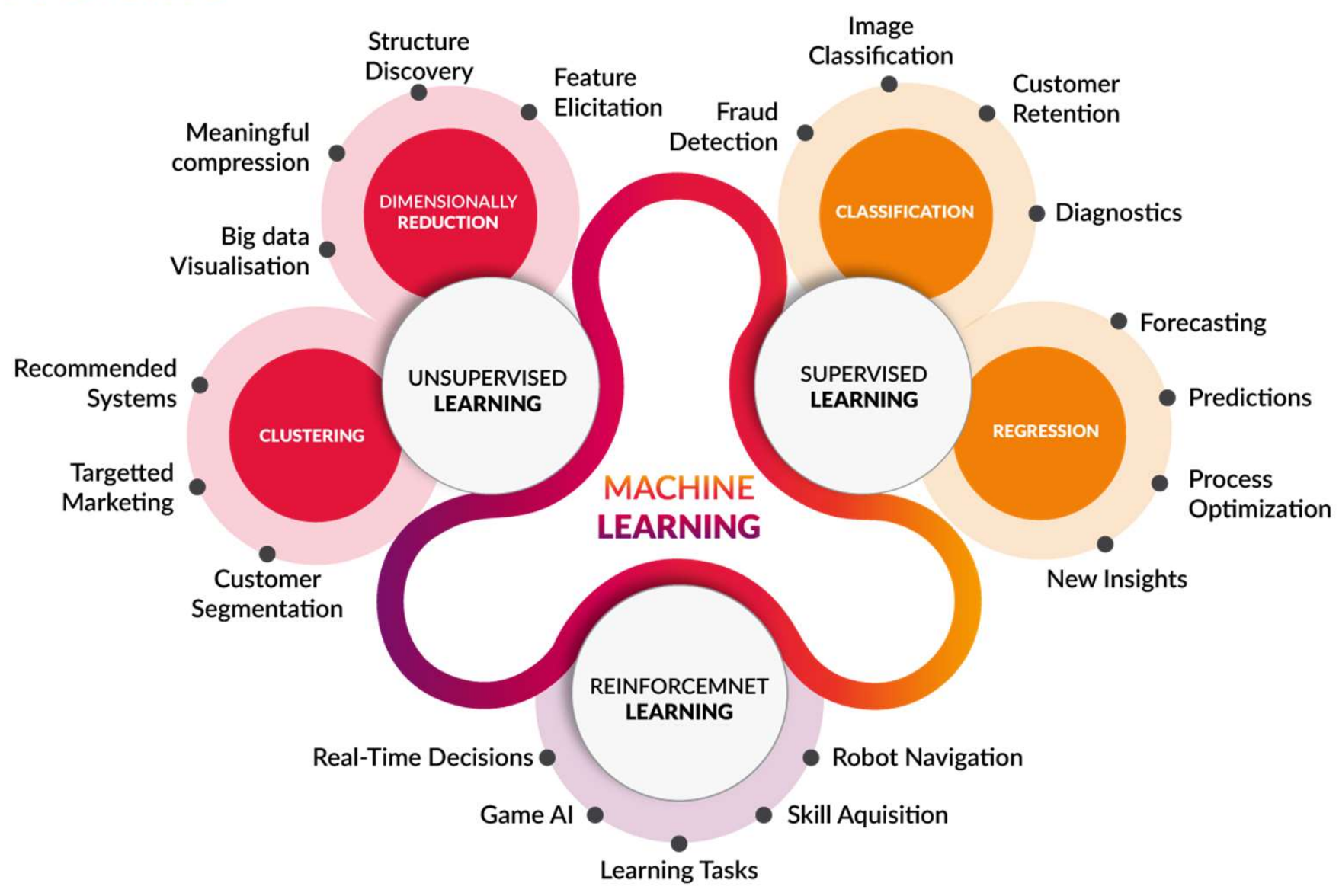

Source: http://www.cognub.com/index.php/cognitive-platform/ 


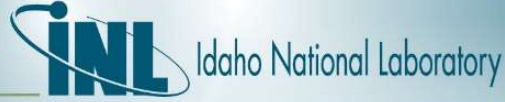

\section{Explainable Al}

The goal of explainable AI

\section{Today}

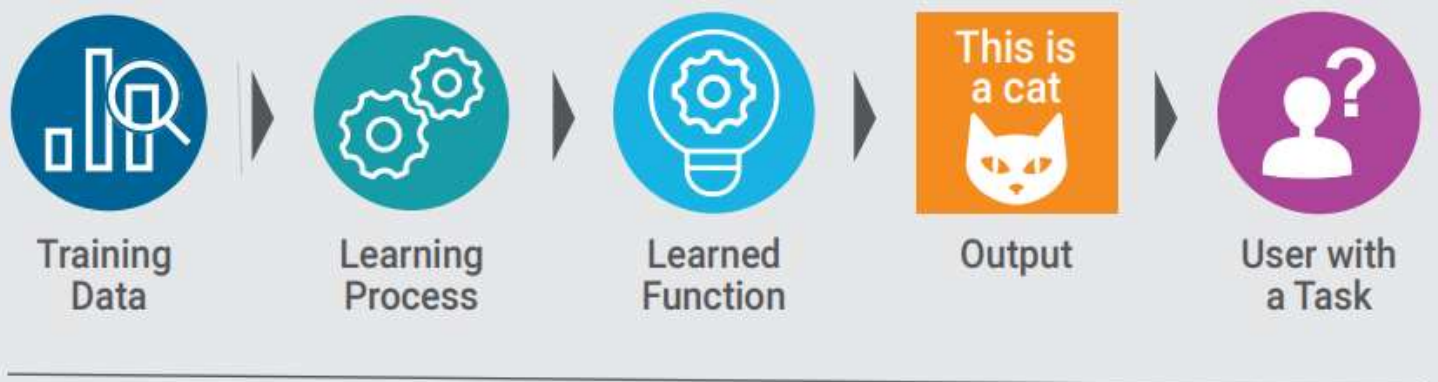

\section{Tomorrow}

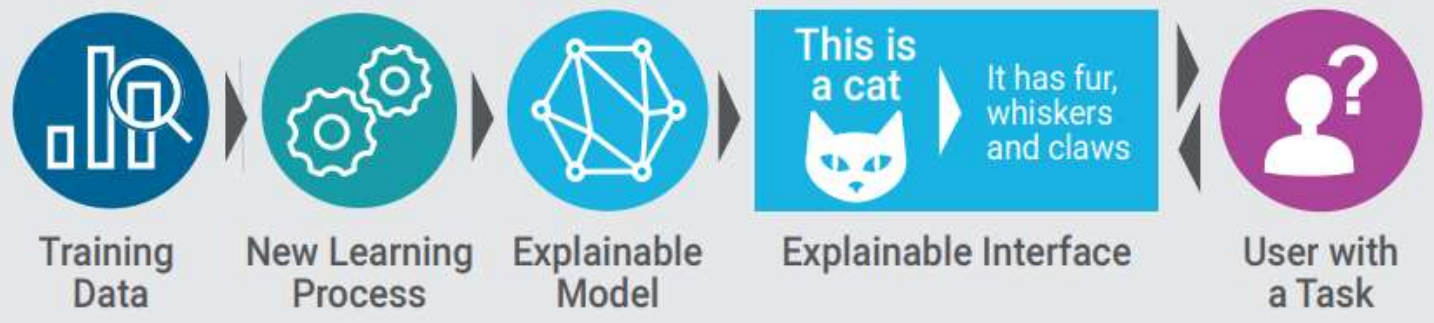

Source: Al and Machine Learning: Key FICO Innovations 


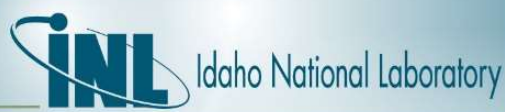

\section{Example Projects}

TAP reactor catalysis machine learning

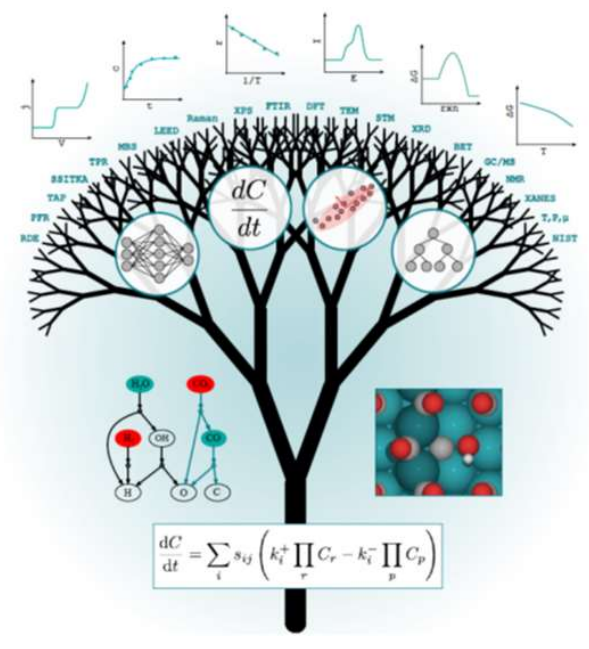

Medford et al. Extracting knowledge from data through catalysis informatics. 2018
Battery life prediction / mechanism estimation

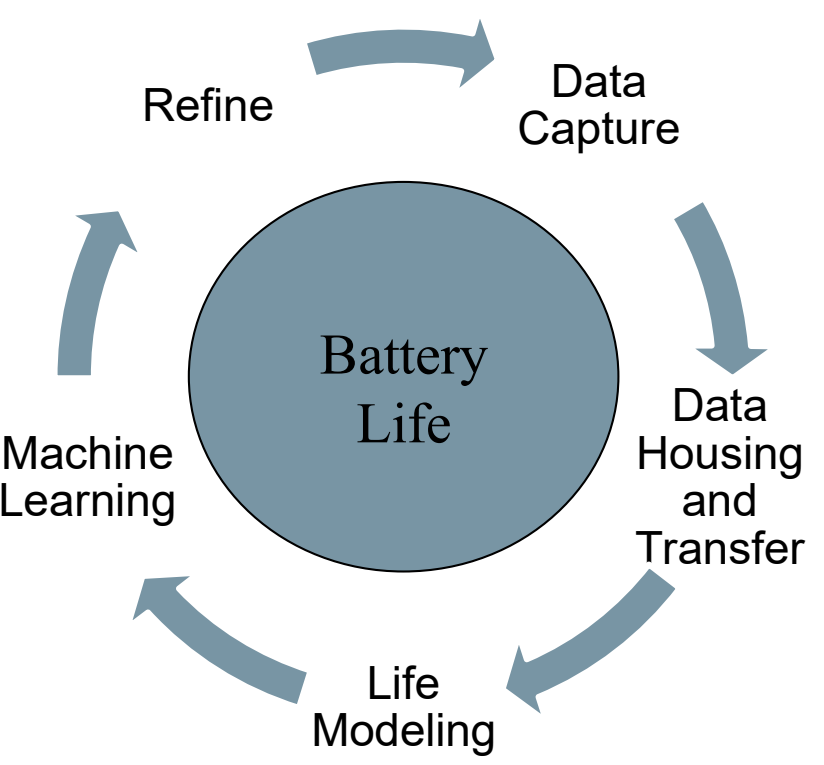

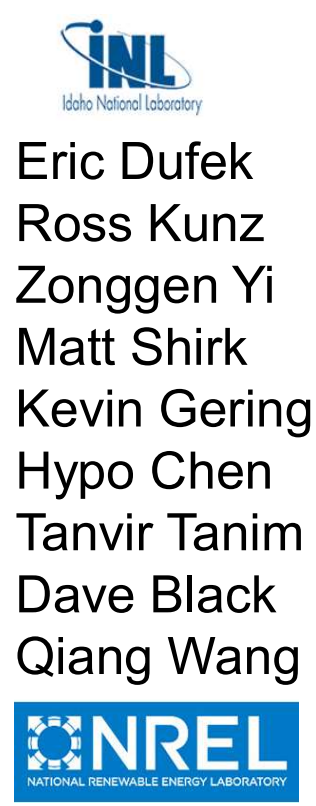

Kandler Smith

Paul Gasper 
IIIIdaho National Laboratory

\section{Questions?}

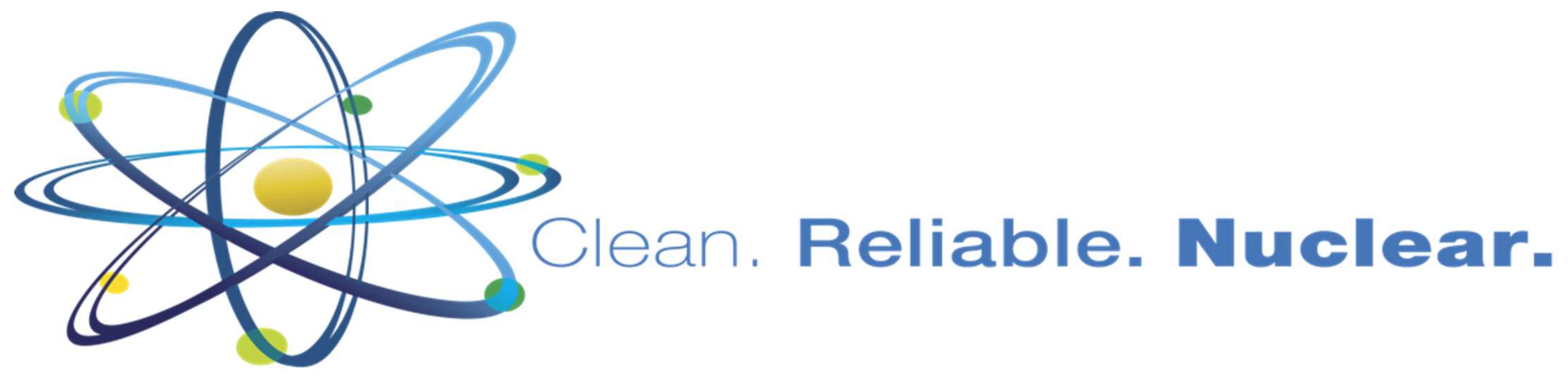




\section{Nancy Lybeck}

Group: Department Manager, Instrumentation, Controls, \& Data Science

Education: Ph.D. in Math from Montana State University.

Fifteen-plus years working with data; 10 at INL

Work focused in: Several projects, including developing a

Risk-Informed Predictive Maintenance Strategy and the

Nuclear Data Management and Analysis System

Presentation Overview

Artificial Intelligence, Machine Learning, and Statistics, Oh My!

- A light-hearted look at the perceived rivalry between data science and statistics. 


\title{
Machine Learning \& Artificial Intelligence Symposium April 17, 2020
}

\author{
Nancy Lybeck, PhD \\ Instrumentation, Controls, \& Data Science \\ Al, ML, and Statistics, Oh My!
}

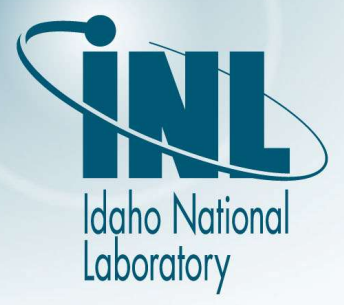


We all love a great rivalry!
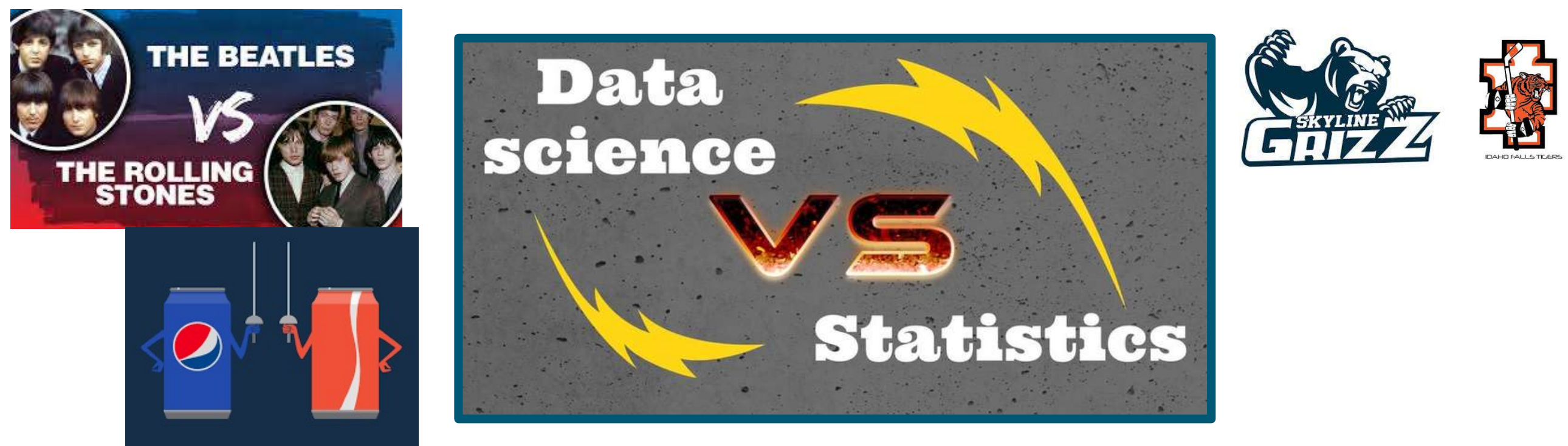

\section{Statistios}
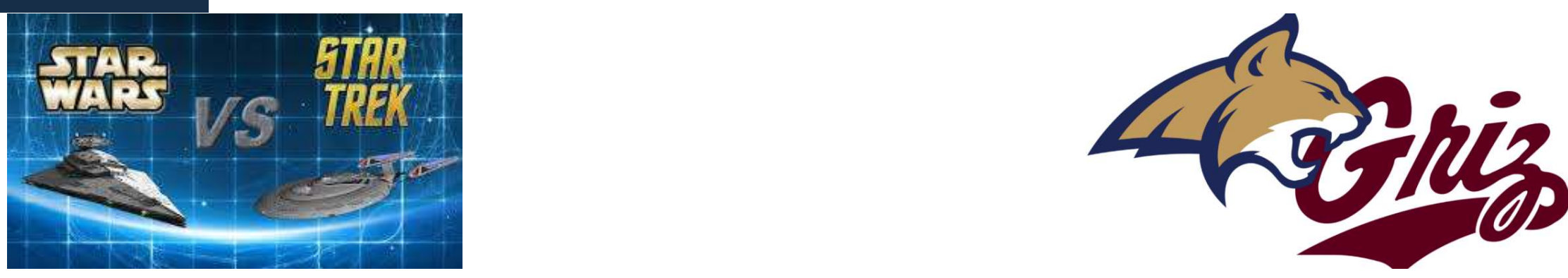


\section{What is Data Science?}

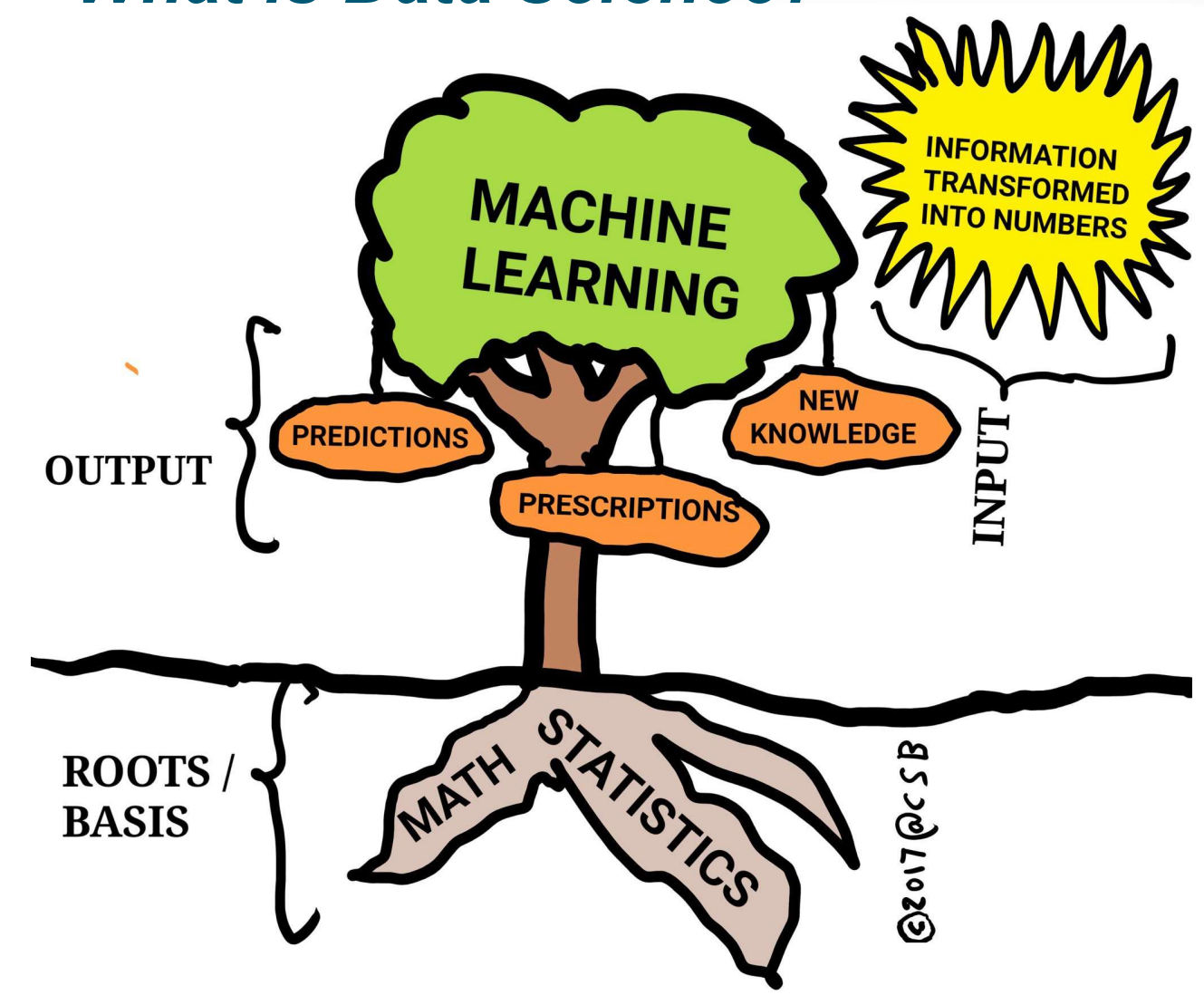

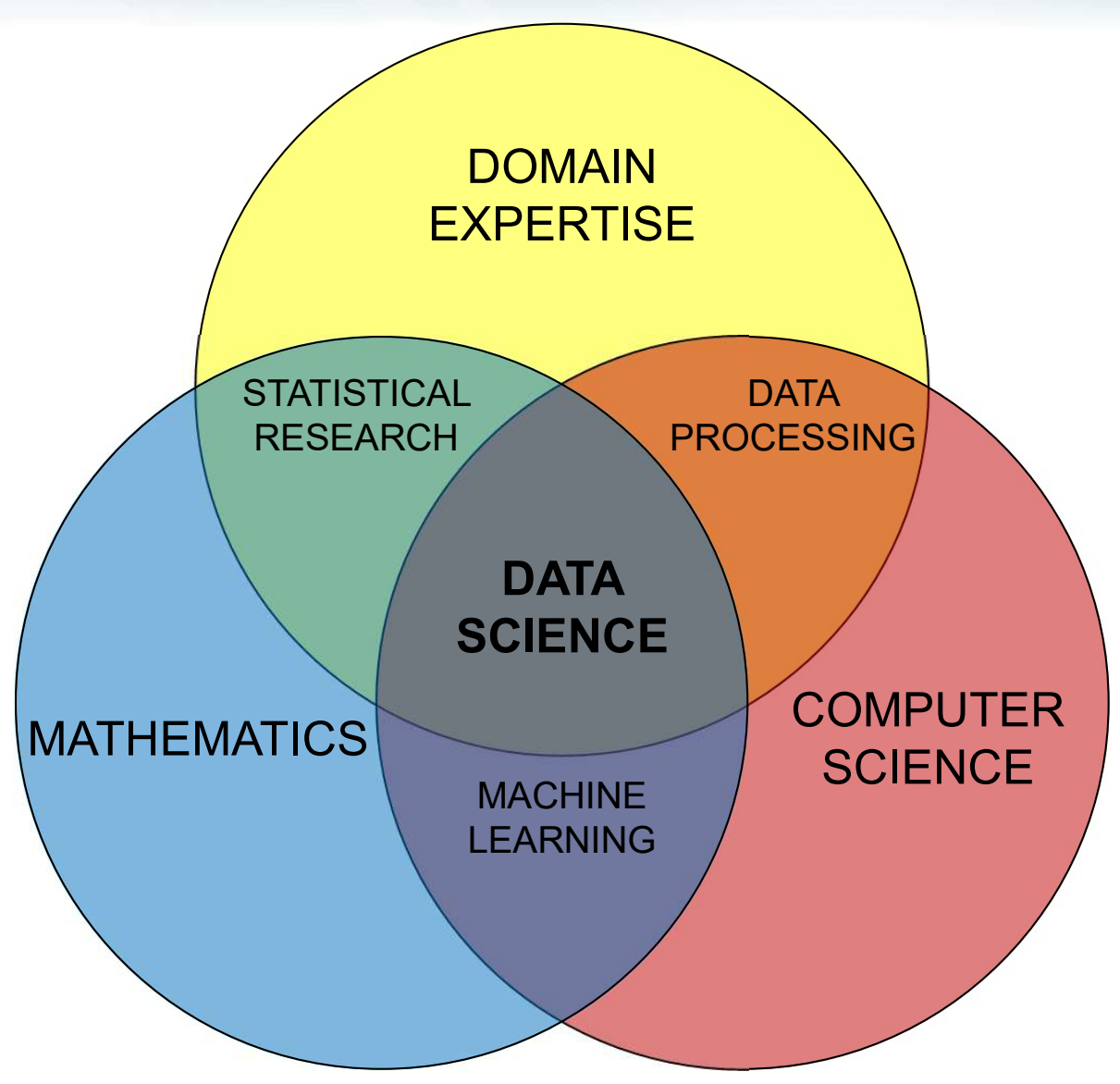

Source: Palmer, Shelly. Data Science for the C-Suite. New York: Digital Living Press, 2015. Print. 


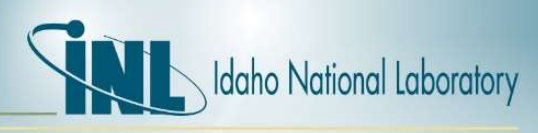

\section{Discussion}

\begin{tabular}{|l|}
\hline \multicolumn{1}{|c|}{ Statistics } \\
\hline - Focus on Inference \\
- Based on probability spaces \\
- models \\
- Often used with tall data \\
- Formalizes understanding of system behavior \\
- Tests a hypothesis about system behavior \\
that a discovered relationship describes a 'true' \\
- effect that is unlikely to result from noise \\
\hline
\end{tabular}

\begin{tabular}{|l|}
\hline \multicolumn{1}{c|}{ Machine Learning } \\
\hline - Focus on Prediction \\
- Based on statistical learning theory \\
- Using general-purpose learning algorithms to find \\
patterns in often rich and unwieldy (nonlinear) data \\
- Particularly helpful with wide data \\
- Makes minimal assumptions about the system \\
- Does not require a carefully controlled experimental \\
- design \\
- of suracy determined with test data set (in the case \\
- Can be difficult to interpret
\end{tabular}

Example from Environmental Science: We might use a statistical model to determine whether a sensor signal response to a certain kind of stimuli is statistically significant, as well as use data from an array of 20 additional sensors to predict the response of the sensor.

The Actual Difference Between Statistics and Machine Learning, Matthew Stewart, 2019. Statistics Versus Machine Learning, Bzdok et al., Nature Methods 15, 223-234 (2018). 
Looking Ahead

- It's all about the data ...

- We need statisticians and data scientists!

- Hold on to the rivalry for fun and for lighthearted teasing, but don't let it get in the way of our ultimate goal: doing great science!

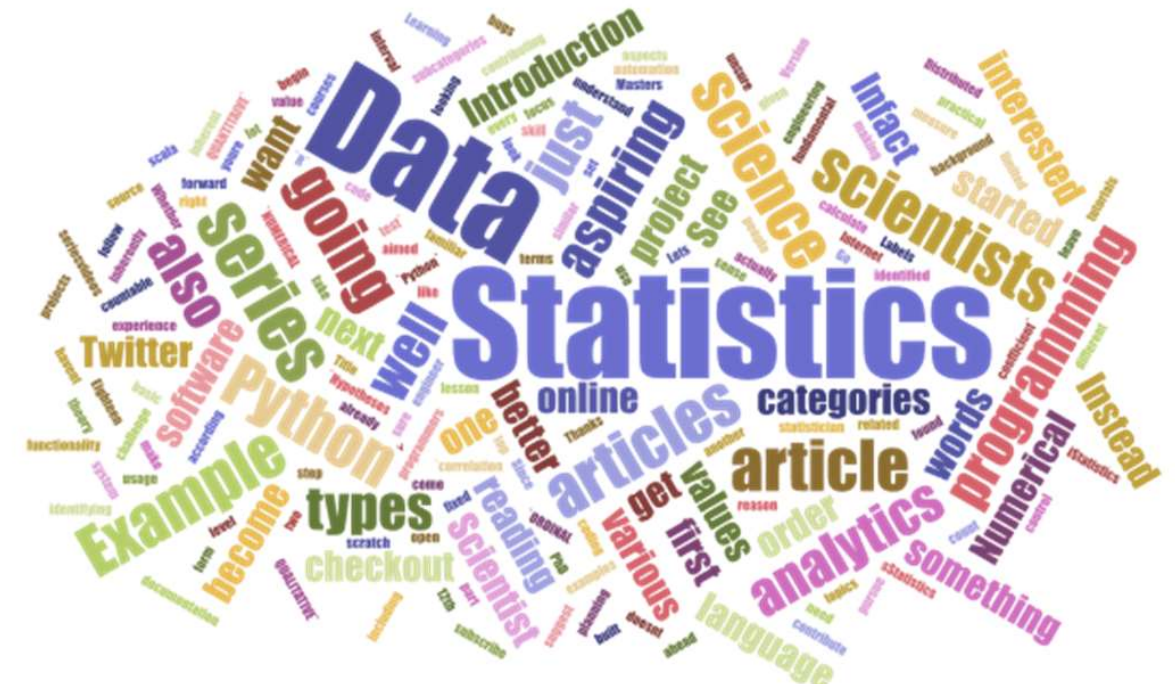


IIIIdaho National Laboratory

Questions?

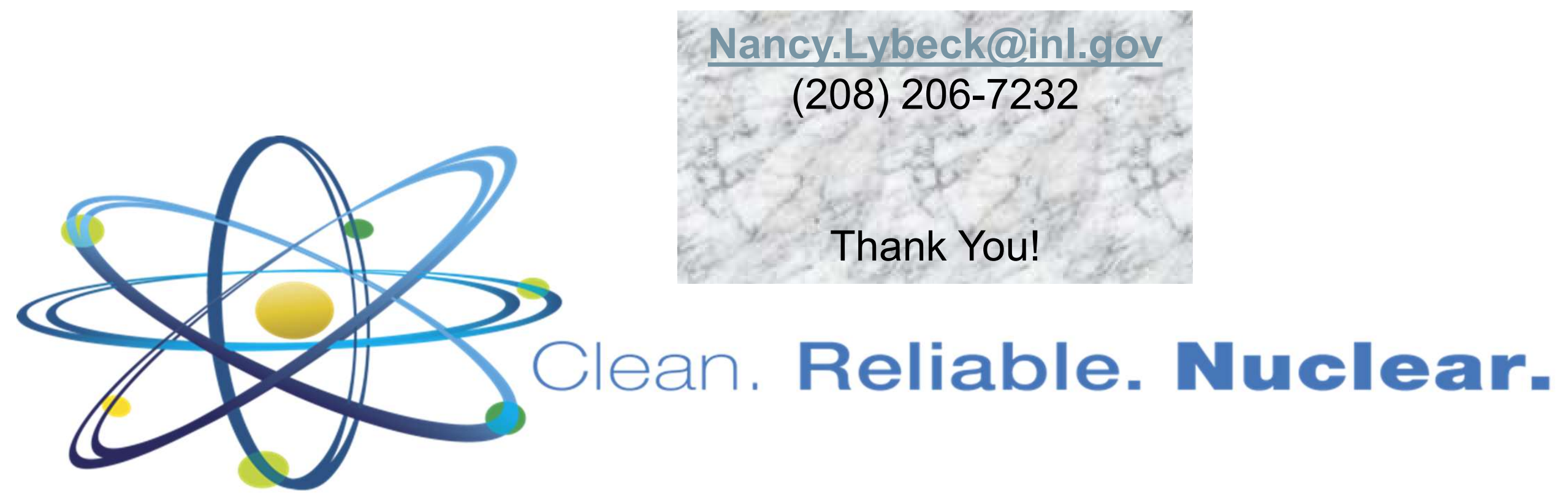




\section{Ronald L. Boring}

Group: Department Manager, Human Factors and Reliability Education: Ph.D. in Cognitive Science from Carleton University Work focused in: Human factors and human reliability

\section{Presentation Overview}

Modeling Human Cognition: It's Not All Machine Learning

- While Al is widely used for industry applications, one of its first uses was to mimic human cognition. The earliest Al techniques were rule based to try to capture the psychology behind human decision making.

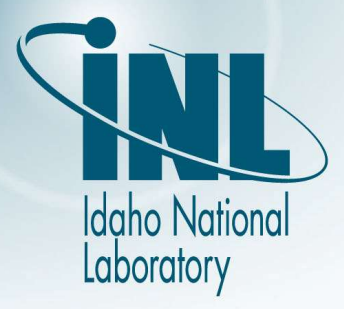




\section{Machine Learning \& Artificial Intelligence Symposium April 17, 2020}

Ronald Laurids Boring, PhD Human Factors and Reliability Dept.

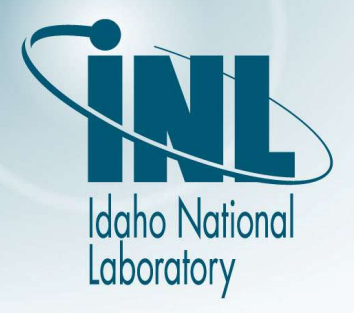

Modeling Human Cognition: It's Not All Machine Learning 


\section{Why Human Cognition?}

1956 Was Watershed Year

- Nuclear History

- Period between USS Nautilus and Shippingport

- Two Congressional Hearings on Automation

- Dartmouth Summer Workshop on Artificial Intelligence

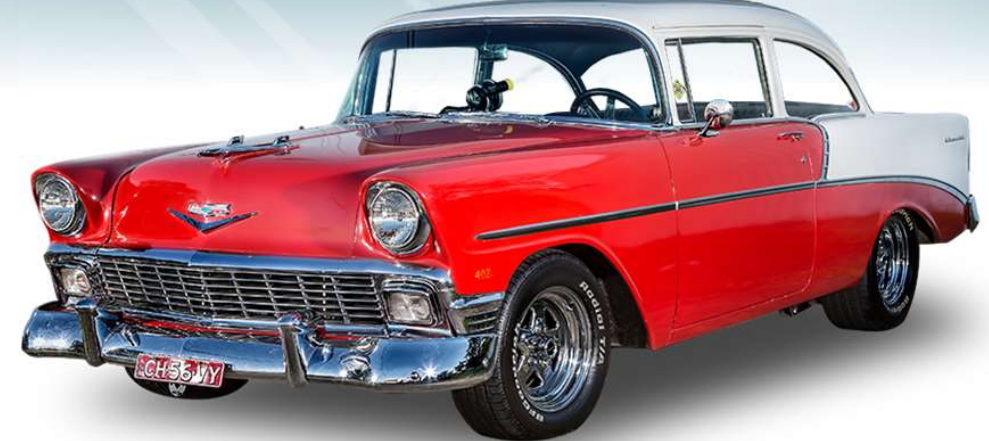

- "We propose that a 2-month, 10-man study of artificial intelligence be carried out during the summer of 1956 at Dartmouth College in Hanover, New Hampshire. The study is to proceed on the basis of the conjecture that every aspect of learning or any other feature of intelligence can in principle be so precisely described that a machine can be made to simulate it."

- Birth of Al, featuring founders like Marvin Minsky, John McCarthy, Claude Shannon, Allen Newell, and Herb Simon

- Symposium on Information Theory at MIT on September 11, 1956

- Birthplace of information processing theory and study of cognition

- Featured George Miller, Noam Chomsky, Allen Newell, and Herb Simon, among others

- Birth of Al and cognitive psychology occurred at the same time, because they were interested in the same problems

- Deconstructing human thinking into information allowed us to make computer models of it 


\section{Why Human Cognition?}

1956 Was Watershed Year

- Nuclear History

- Period between USS Nautilus and Shipp

- Two Congressional Hearings on Automatio

- Dartmouth Summer Workshop on Artificial

- "We propose that a 2-month, 10-man stu summer of 1956 at Dartmouth College in on the basis of the conjecture that every can in principle be so precisely describec sensory registration

- Birth of Al, featuring founders like Marvin Newell, and Herb Simon

\section{Basic Information Processing Model}

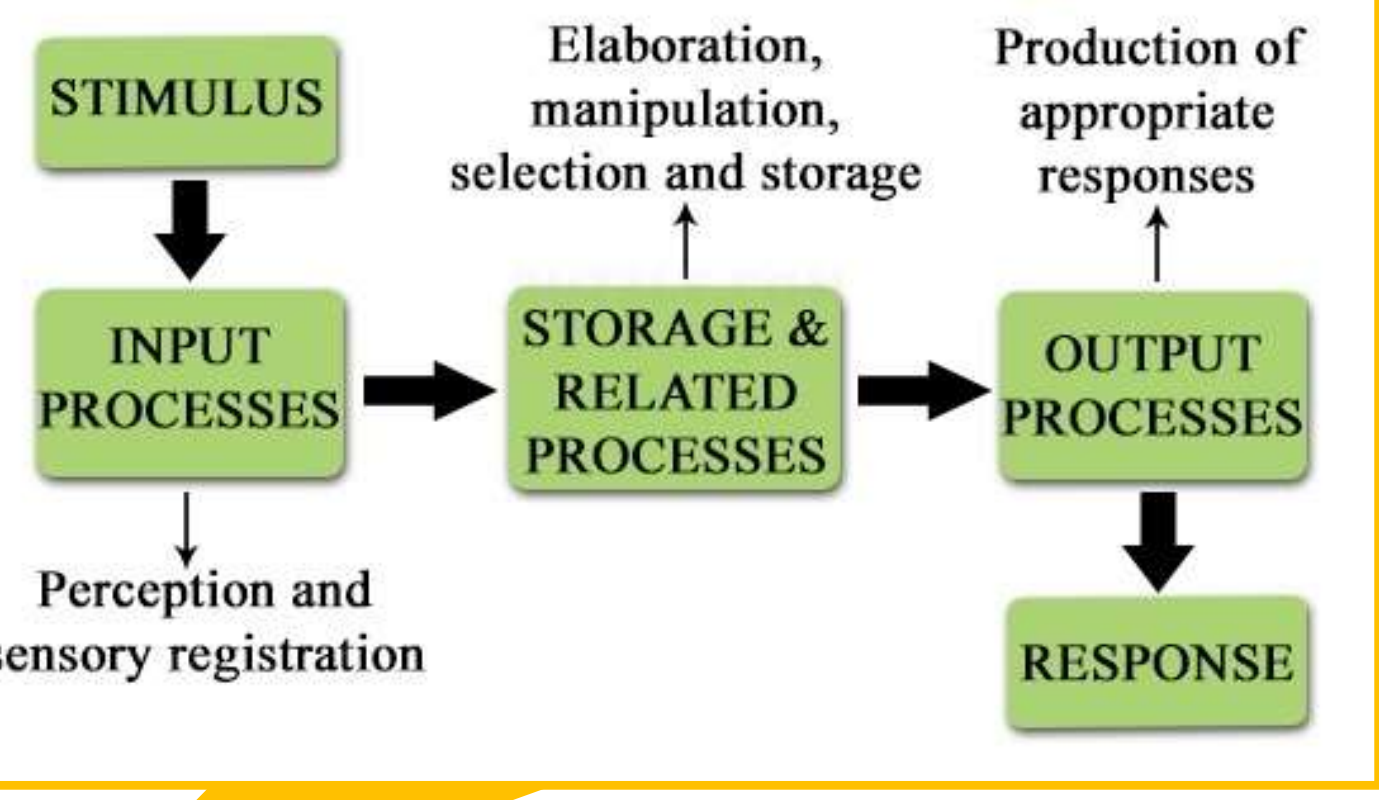

- Symposium on Information Theory at MIT on Sep

11,1956

- Birthplace of information processing theory ? study of cognition

- Featured George Miller, Noam Chomsky, Allen Newell, and Herb Simon, among others

- Birth of Al and cognitive psychology occurred at the same time, because they were interested in the same problems

- Deconstructing human thinking into information allowed us to make computer models of it 


\section{Al is More Than Machine Learning}

\section{Two Types of Al}

- Good Old-Fashioned Al (GOFAl)

- Symbolic logic systems to represent basic elements of human thought like language, numbers, or goals

- Expert systems featuring if-then logic

- General Problem Solver created by Newell and Simon in 1959

- Much of focus was not to create learning but to capture human-like intelligence

- Neural Networks

- Perceptron developed in 1958 as approximation of single-cell neuron

- By 1960s, mathematical algorithms like backpropagation developed to allow perceptrons to learn through training

- Machine learning

- Multiple perceptrons chained together to create neural networks

- More layers of neural networks chained to together to create deep learning

- Different Uses

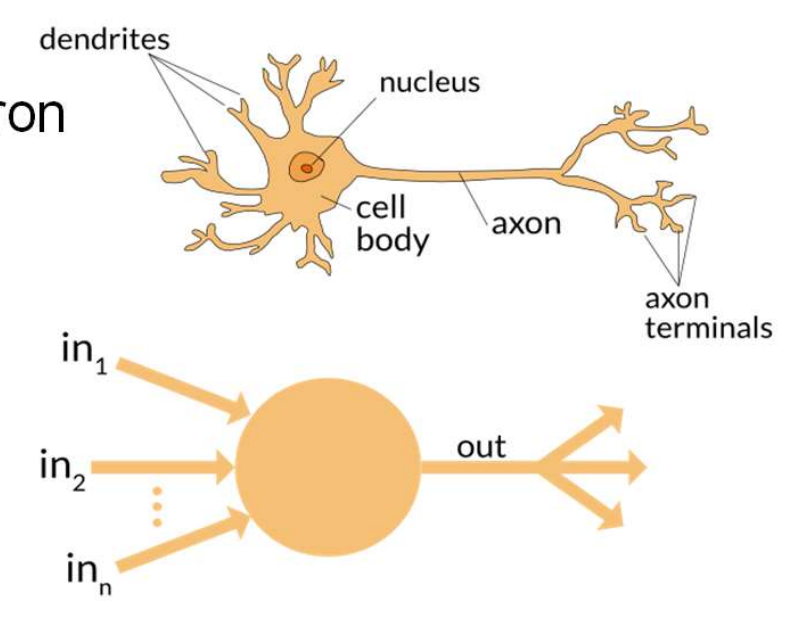

- GOFAl is good at following rules and making decisions

- Neural networks are good at pattern recognition when trained 


\section{Why is Human Cognition Relevant to Al?}

Humans Are Better At-Machines Are Better At (HABA-MABA)

- Humans are (still) better at some things

- Generalization and flexibility

- Judgement and decision making

- Responding to novel events and degraded conditions

- Creativity and problem solving

- Sentience and consciousness

- Machines are better at some things

- Performing routine, repetitive, or precise tasks like monitoring

- Multitasking

- Quick responses
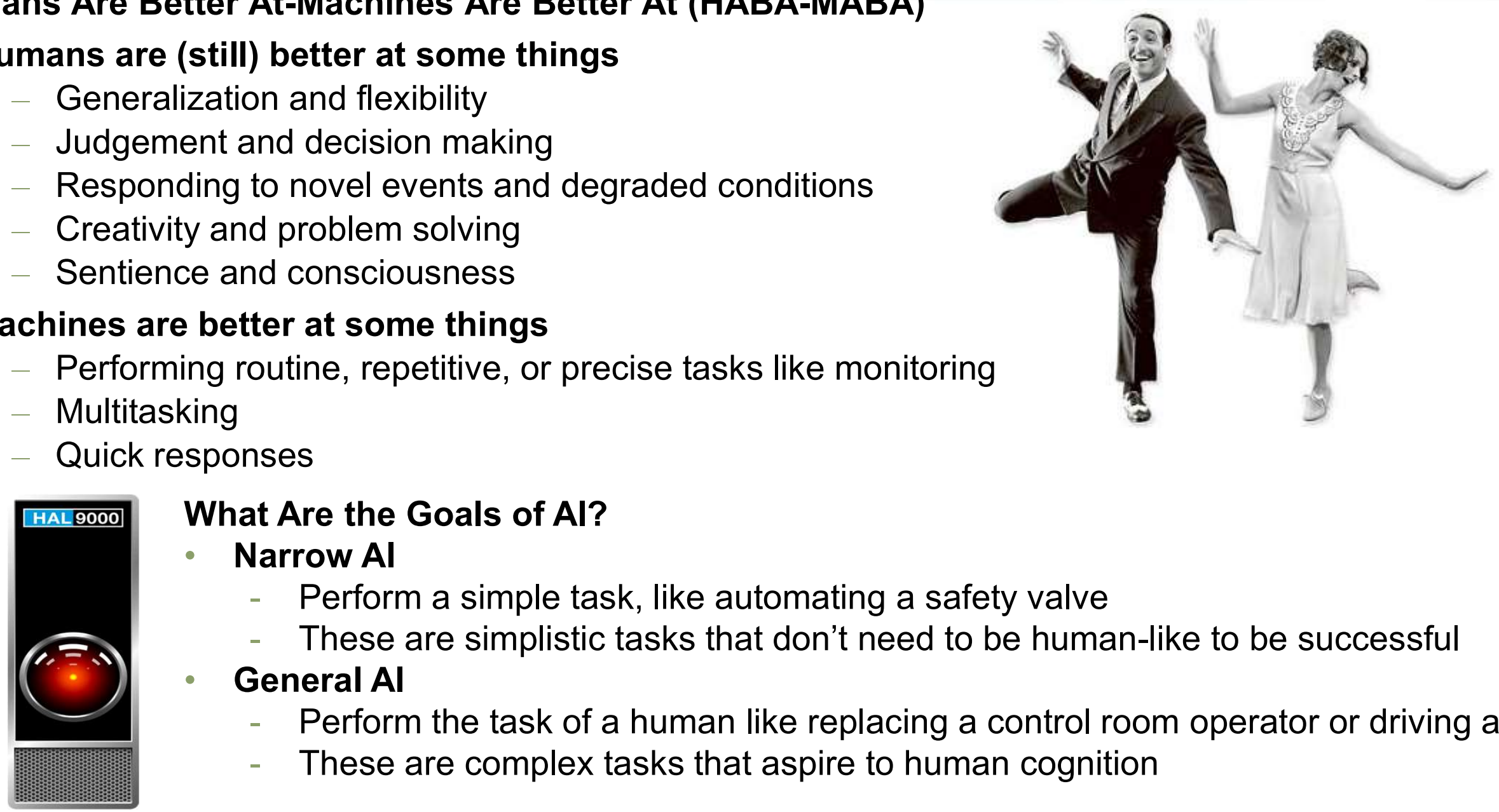

What Are the Goals of Al?

- Narrow Al

- Perform a simple task, like automating a safety valve

- These are simplistic tasks that don't need to be human-like to be successful

- General Al

- Perform the task of a human like replacing a control room operator or driving a car

- These are complex tasks that aspire to human cognition 


\section{The Future of Cognition and Al}

\section{Principles for the Intersection of Humans and Al}

1. $\mathbf{A l}=$ Knowledge + Learning

- To say someone is intelligent does not mean they are good learners, it means that they are knowledgeable

- $\mathrm{Al}$ is a mix of GOFAl (knowledge) and neural networks (learning)

- It takes both to create something like autonomous vehicles: see the road + follow the rules

2. Machine Learning Has Limits

- We think of ML as producing superintelligence, but most applications are really narrow Al

3. Humans are the Users of Al

- Sometimes we seek not to replace the human but enhance or complement them (e.g., predictive maintenance)

- Need to develop explainable Al that humans can understand and work with

- How does regulator approve Al for safety applications like nuclear when Al isn't transparent in what it's doing?

- Data visualization-representing patterns out of complexity-is one form of usable Al

4. Humans are Big Data

- Human performance and knowledge can still be harvested to improve Al 


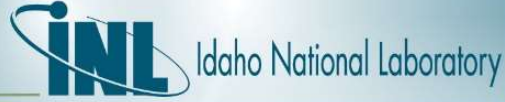

\section{Questions?}
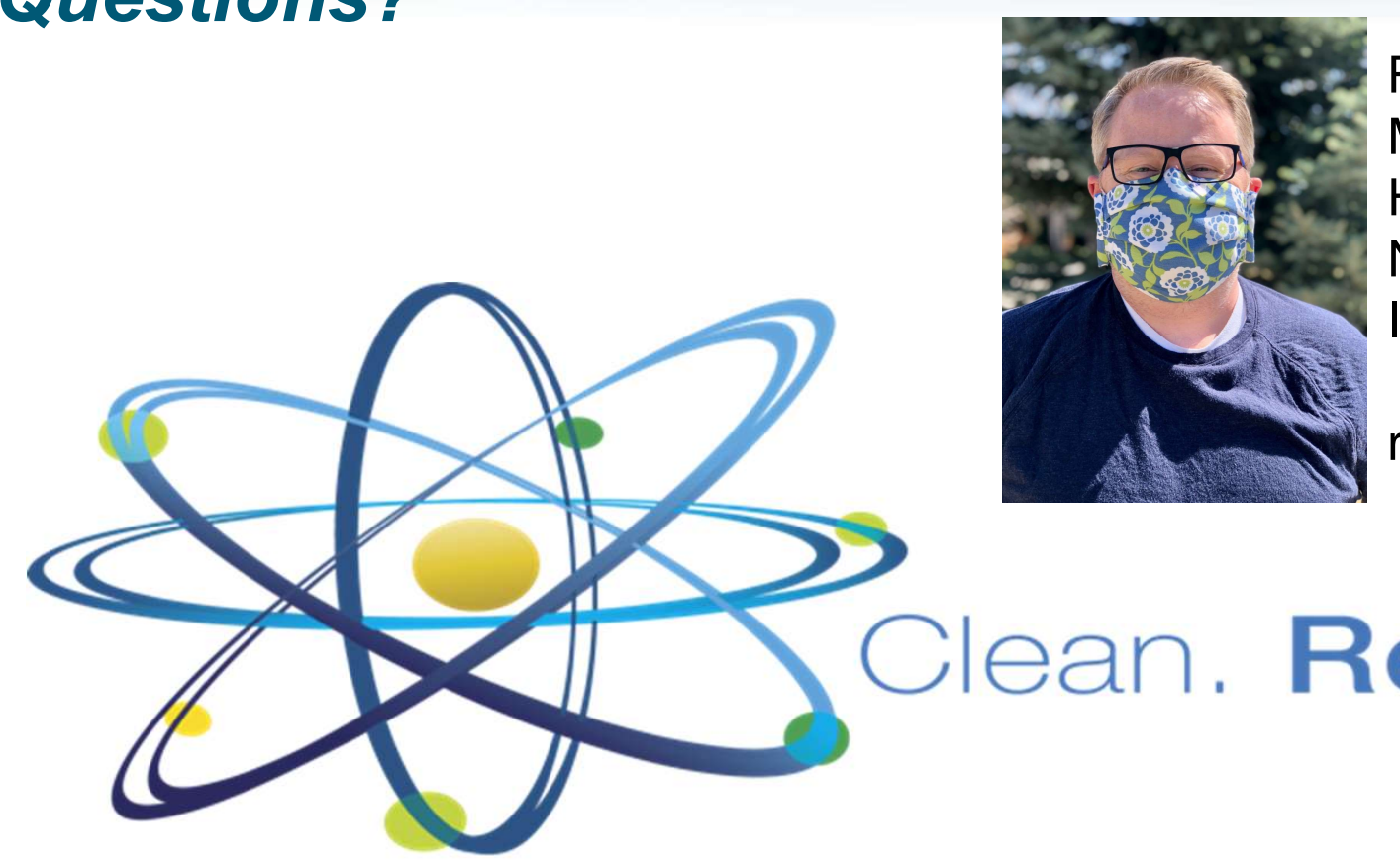

Ron Boring, $\mathrm{PhD}$

Manager \& Distinguished Scientist Human Factors \& Reliability Department

Nuclear Safety and Regulatory Research Division Idaho National Laboratory

ronald.boring@inl.gov

\section{Reliable. Nuclear.}




\section{Humberto E. Garcia}

Group: Systems Science \& Engineering Education: PhD

Work focused in: Extensive experience in advanced systems methods for the design, integration, optimization, and operation of cyber-physical systems (CPS)

Presentation Overview

Secure Embedded Intelligence (SEI) in Smart Nuclear Systems

- Research needed / Gaps for implementing SEI in Smart Reactor Systems

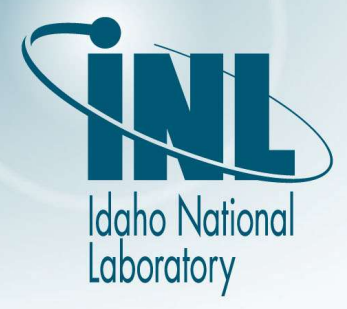




\section{Secure Embedded Intelligence (SEI) in Smart Reactors}

Topics: multi-scale, multi-layered computing, hybrid physics-based, datadriven M\&S, digital twins (DT), integrated state awareness (ISA), adaptive observation \& actuation, intelligent controls, automated reasoning, digital assets

Humberto E. Garcia, PhD Cyber-Physical Systems Integration, Optimization \& Resilient Controls
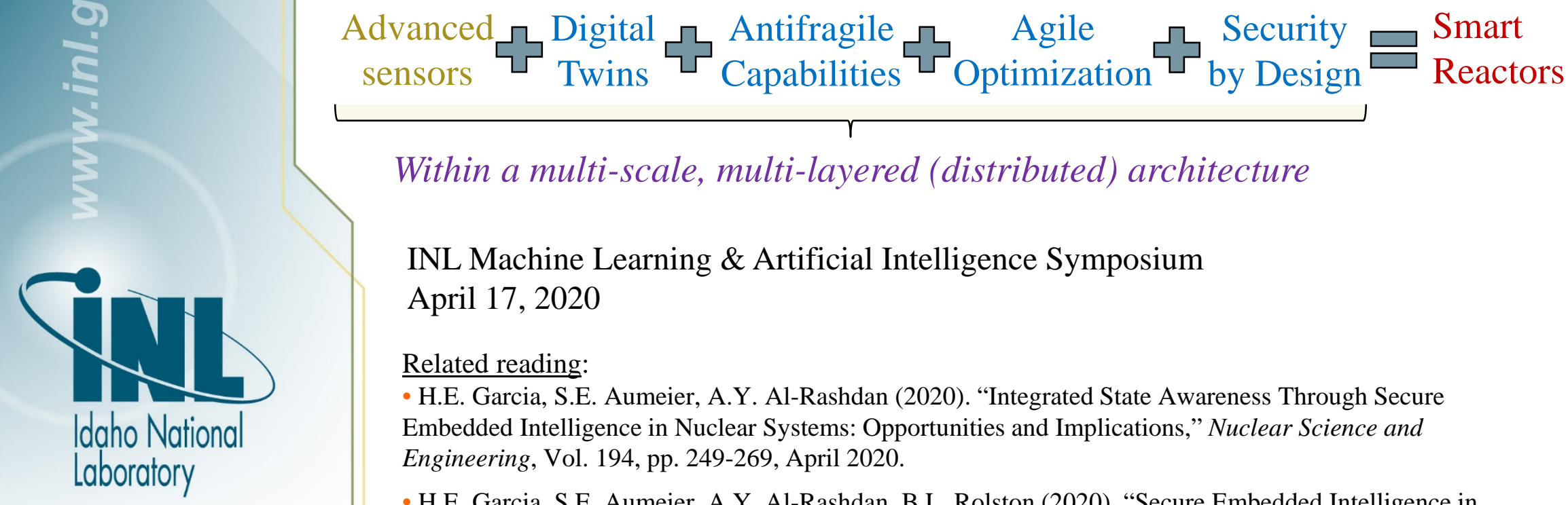

Within a multi-scale, multi-layered (distributed) architecture

INL Machine Learning \& Artificial Intelligence Symposium April 17, 2020

\section{Related reading:}

- H.E. Garcia, S.E. Aumeier, A.Y. Al-Rashdan (2020). "Integrated State Awareness Through Secure Embedded Intelligence in Nuclear Systems: Opportunities and Implications," Nuclear Science and Engineering, Vol. 194, pp. 249-269, April 2020.

- H.E. Garcia, S.E. Aumeier, A.Y. Al-Rashdan, B.L. Rolston (2020). "Secure Embedded Intelligence in Nuclear Systems: Framework and Methods,” Annals of Nuclear Energy, Vol. 140, 2020, 107261. 


\section{Why it is important to industry}

- Operations \& maintenance (O\&M) cost reduction \& simplification

- Economics (e.g., $15-50 \%+$ fixed O\&M cost reduction)

- Real-time asset condition assessment

o from preventive to predictive

o Predictive maintenance (PdM), proactive asset performance/health management (APM)

o Early anomaly/health detection, diagnostics \& prognostic of systems, structures, components (SSC)

- Improved reliability, availability, maintainability, safety, security

- Market expansion, application flexibility, nuclear industry sustainability

- Flexible operation

- Remote and transportable deployments

- Broad range of "plug-and-play” (commercial and emergency) applications

- Design and operations margin reduction and optimization

- Simplicity and uncertainty \& imprecision tolerance

- Unprecedented system-state knowledge enabling:

- Adaptive control (e.g., idle, startup, shutdown), automated reasoning, decision-making

- Recognition \& classification of abnormal and degradation signatures

- Inherent, proactive cybersecurity and cyber-defense by design

- $\quad$ Real-time metric (e.g., risk) quantification, optimization, management

- Human reliability and productivity enhancement

- Integrated, precision data availability and presentation / visualization 


\section{Current trends in diverse industries}

Vehicles w/ limited “automated processing”
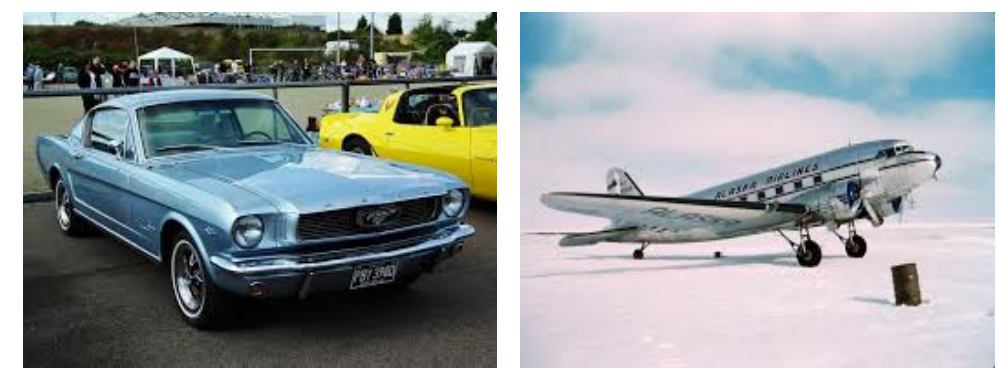

“Labor-intensive” manufacturing

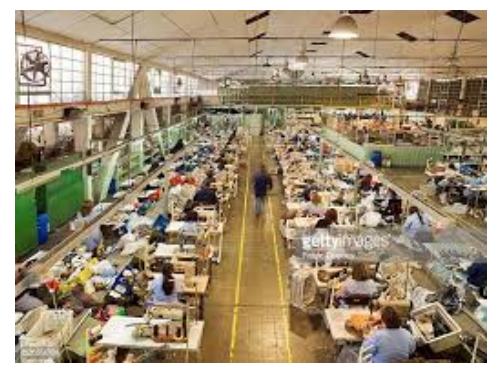

Autonomous "smart" vehicles
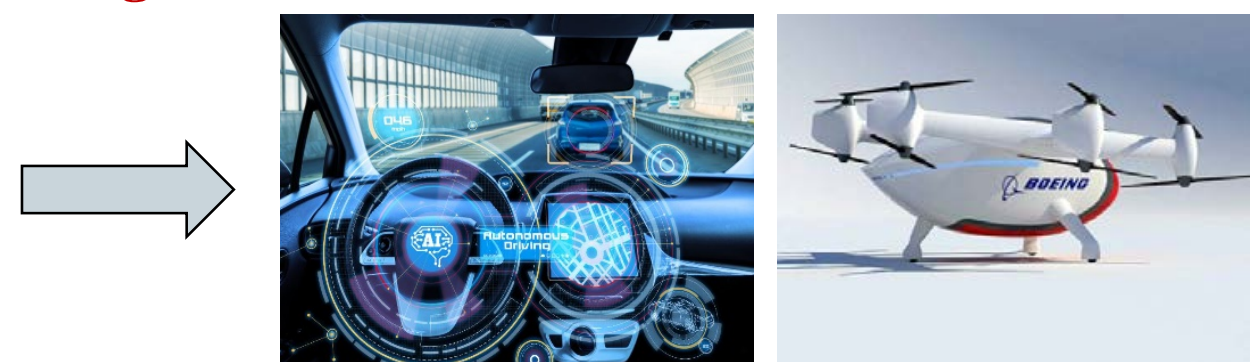

Autonomous “smart” manufacturing

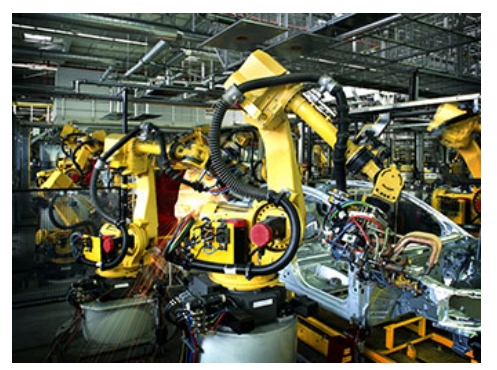

Is autonomy of smart reactors the goal ? or rather to identify fundamental attributes a system should be equipped with to meet desired (smart) functionalities (e.g., autonomy) ?

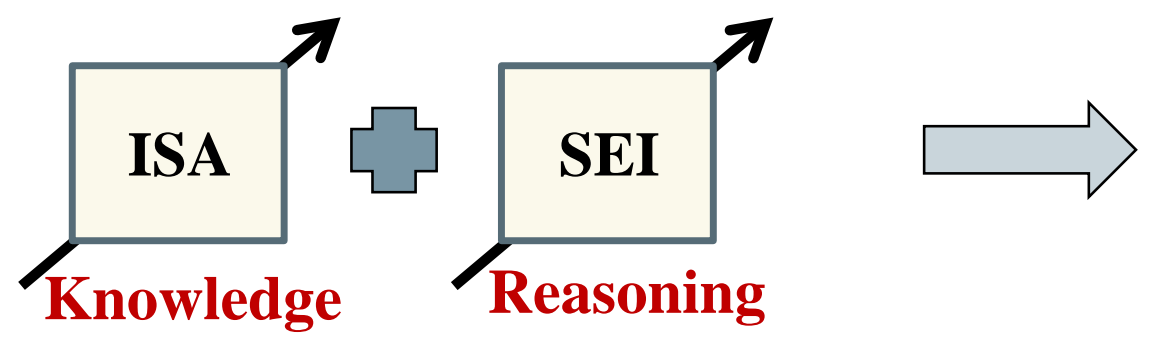

To achieve "smart" functionalities (e.g., autonomy, asset health assessment)

SEI: Secure embedded intelligence

ISA: Integrated state awareness Design for optimal levels of ISA \& SEI to achieve objectives 


\section{Phased implementation of SEI-ISA in advanced nuclear systems}

\section{Add fundamental capabilities}

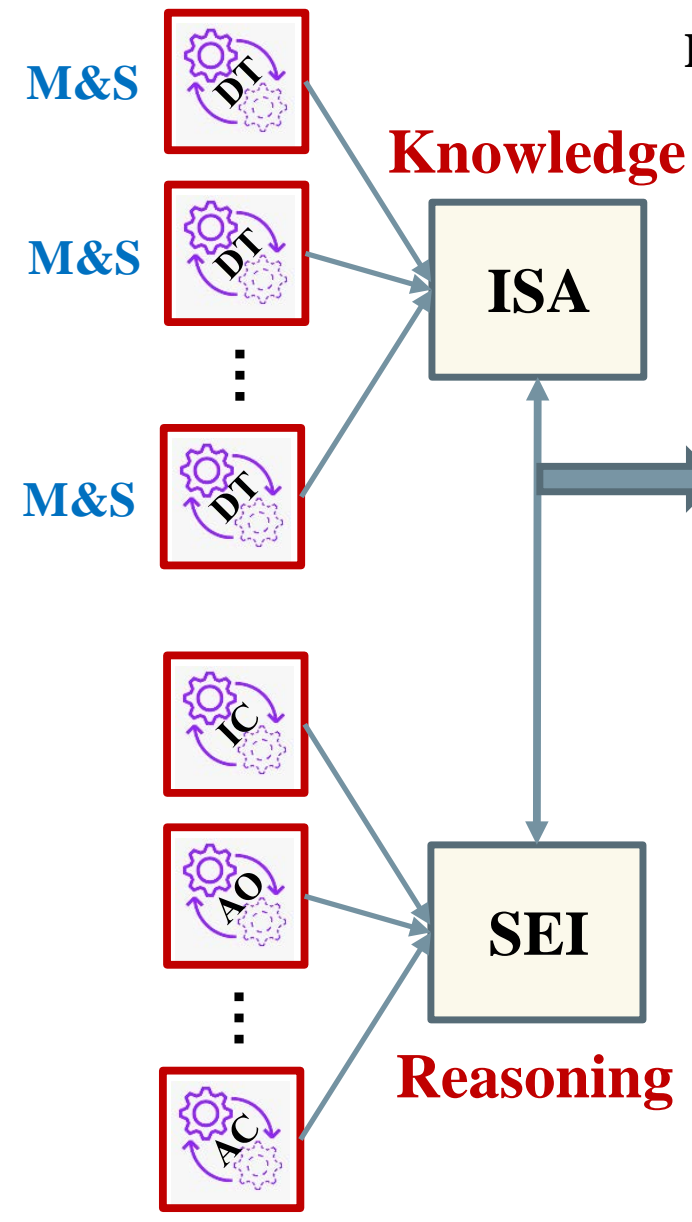

DT: Nested Digital Twin (model-based + data-driven, multiscale, multilayered)

\section{To achieve fundamental functionalities}

- Estimate (e.g., current system state)

- Predict (e.g., future system state)

- Understand (e.g., consequences of stressors, actions)

- Learn (e.g., relationships from observed patterns)

- Decide “optimal” paths forward (e.g., control actions)

\section{Disruptive advances $\quad \square \quad$ Disruptive potentials}

- multi-scale / multi-layered computing $\checkmark$ Cost (HPC \& edge computing)

- $\quad$ physics-based, data-driven hybrid M\&S and analysis

- multi-layered adaptive observation \& actuation

- intelligent controls (IC) \& supervision

$\checkmark$ Simplicity

$\checkmark$ Flexibility

$\checkmark$ Systems optimization

$\checkmark$ Inherent security, resiliency

$\checkmark$ System-state transparency

- agile optimization (AO)

- AI-enhanced capabilities (AC) 


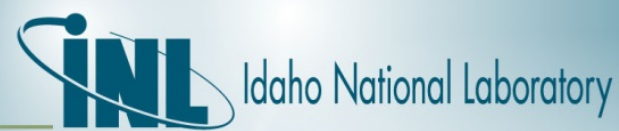

Intelligent nuclear assets: Multi-scale, multi-layered integration of advanced monitoring, control \& supervision (MCS) functions

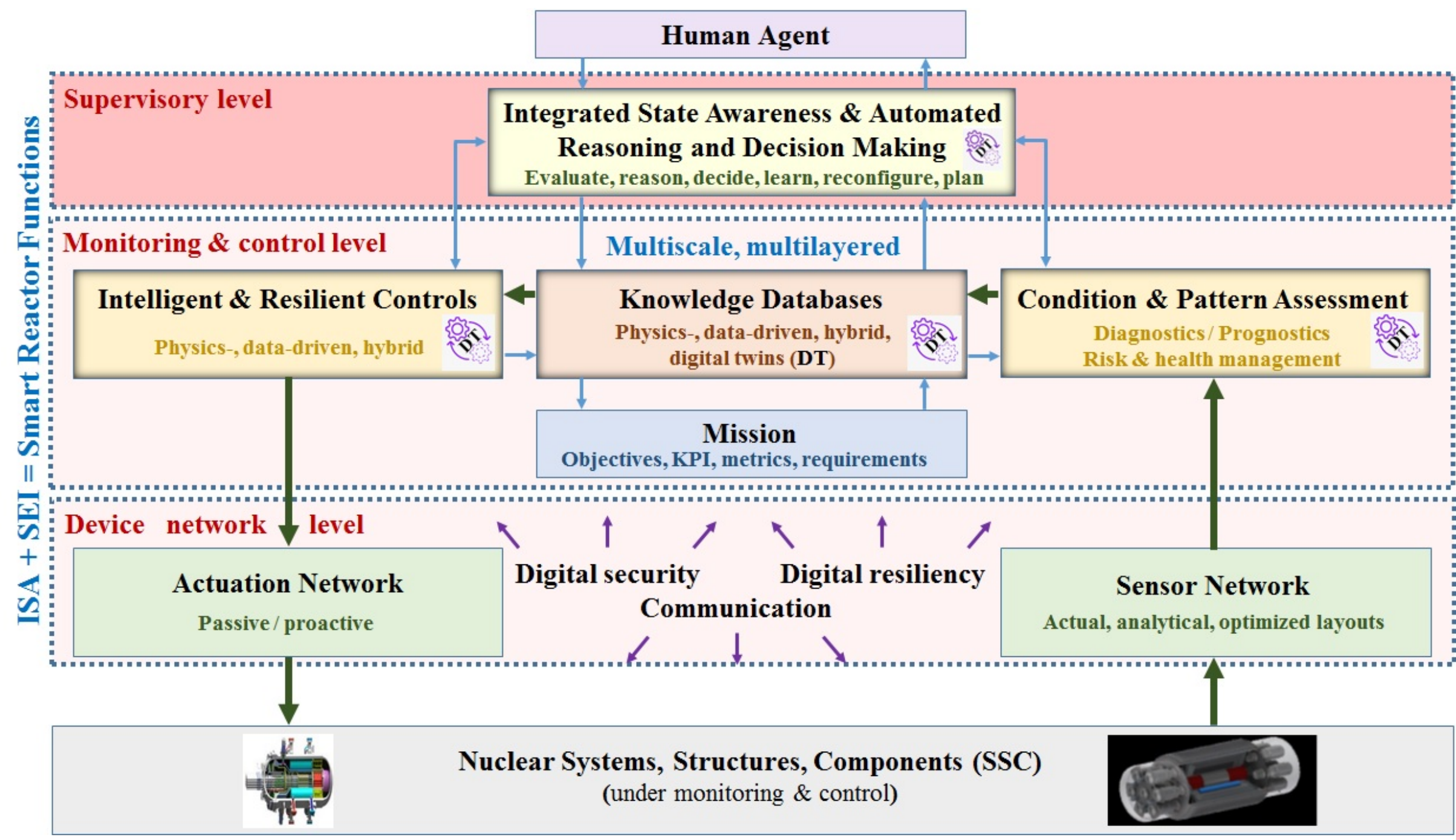




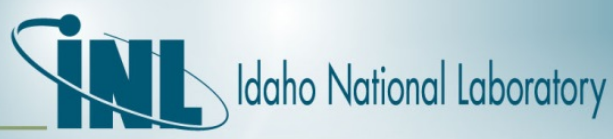

\section{Research opportunities for implementing SEI in smart reactor systems}

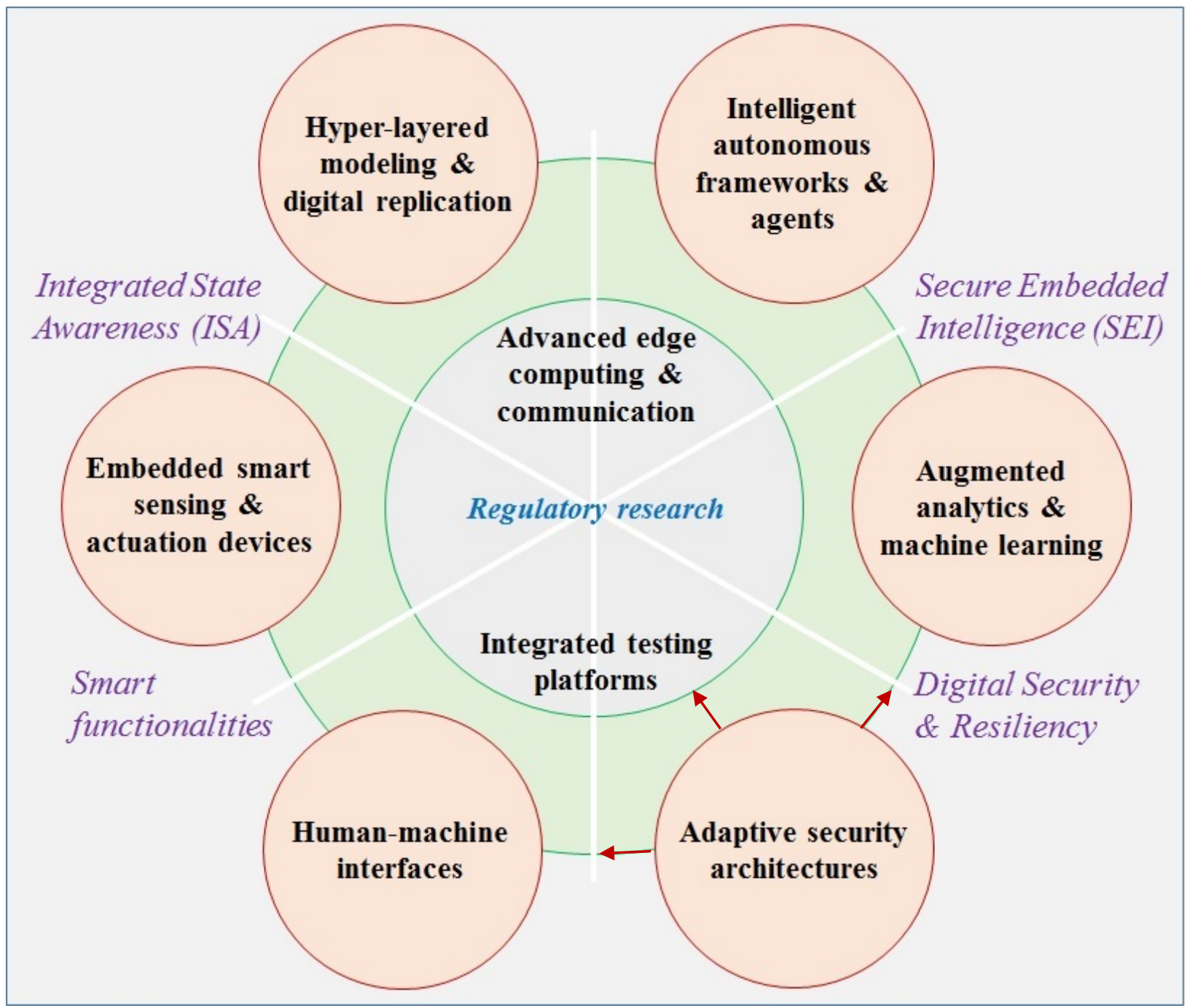

\section{Products}

- Architectures

- Frameworks

- Information infrastructures

- (edge-, system-) methods, models, agents, algorithms

- Hardware / software capabilities and devices

- Design impacts

- Testbeds

- Pilots

- Standards

- Policies 


\section{Idaho National Laboratory}

\section{Questions?}

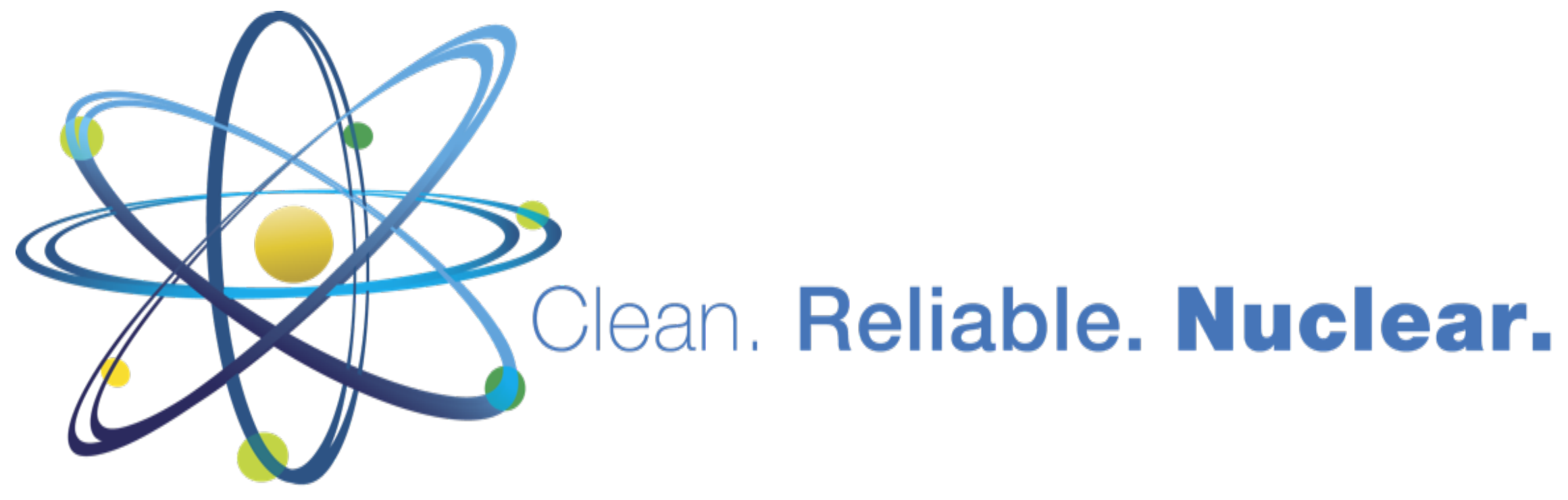




\section{Victor G. Walker}

Group: Mobility Systems and Analytics

Education: B.S. and M.S. degrees in Computer Science with a focus on intelligent and adaptive systems and worked for 11 years at IBM before joining INL

\section{Presentation Overview}

Al in Robotics and Applying Natural Connections

- Al in Robotics has some unique characteristics. It involves an intelligent system that interacts with the real world and these issues can influence both how a system learns and what we expect from the systems. A key goal is creating a system that allows us to use robotics as a natural partner. 


\title{
Machine Learning \& Artificial Intelligence Symposium April 17, 2020
}

\author{
Victor Walker \\ Advanced Transportation \\ Al in Robotics and Applying Natural \\ Connections
}

Idaho National 


\section{Robotics \\ (What is it?) \\ Computation \\ Mobility}

Humanoid Robots

Unmanned Aerial Vehicles (UAV) Unmanned Ground Vehicles (UGV) Self-Driving Cars Robotic Arms
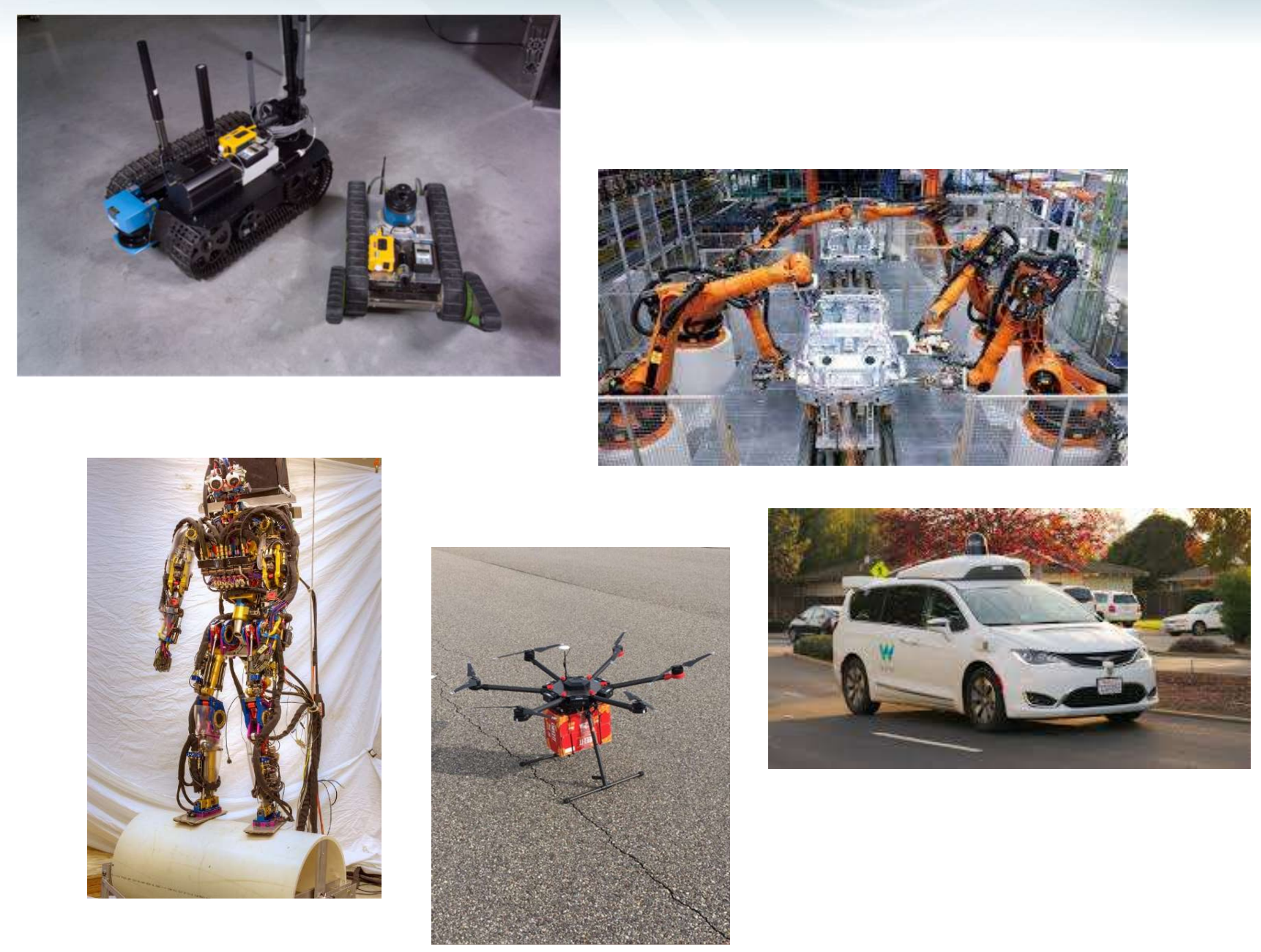


\section{Robotics and Intelligence (Introduction)}

\section{Intelligence}

(What is it in Robotics?)

Behavior-based

Does it "Act" intelligently?

Does it do intelligent tasks?

Does it partner well?

Needs:

\section{Sensors}

Tasks

Training
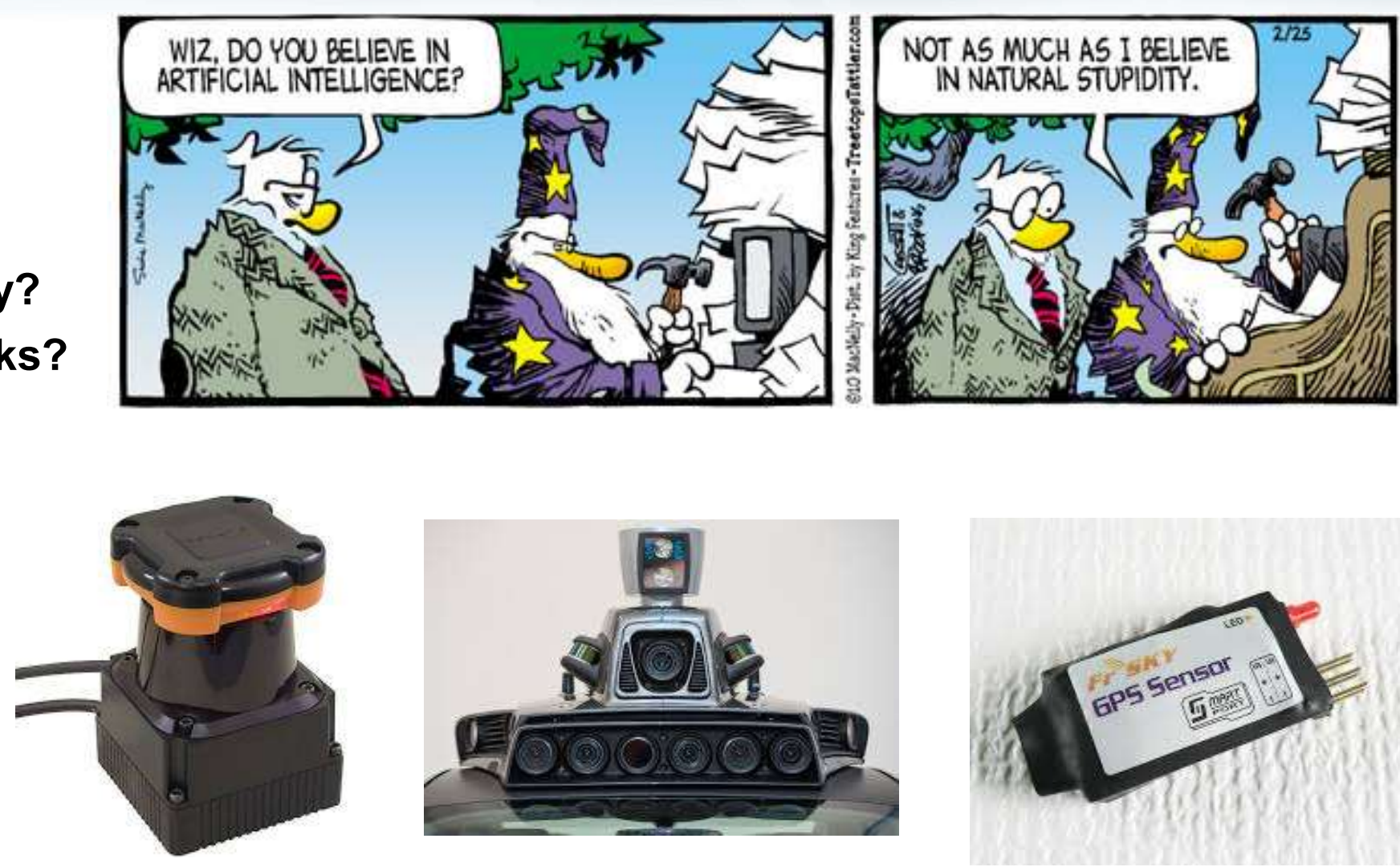


\section{Robotics (Relevance)}

\section{Intelligent Robotics enables a brave new world....}

Robotics enables a broad range of tasks

Dangerous

Precision

Repeatable

Dull

Efficient

Remote

Intelligence enhances Partnership

Partner with humans on tasks.

Change the world... based on location

Understand environment / Aid decisions

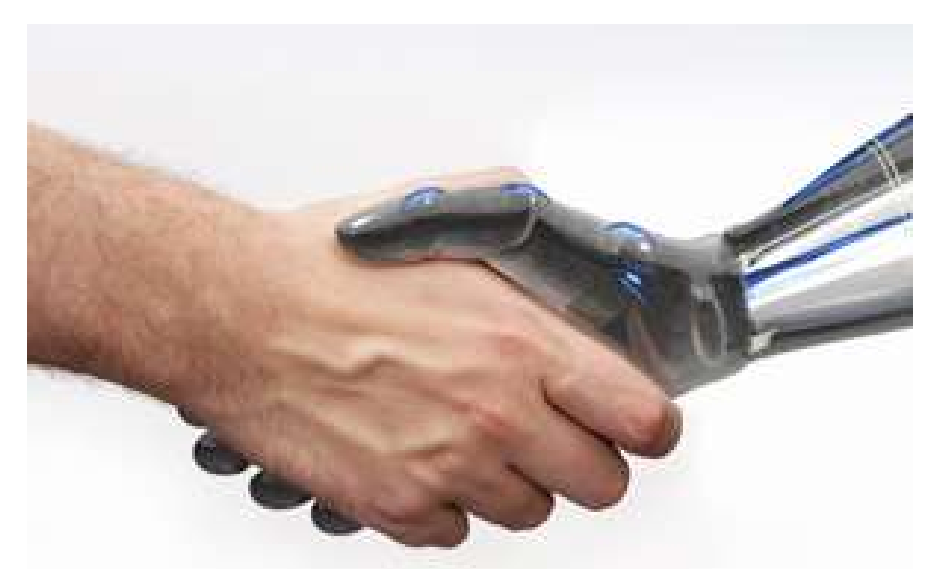

Look for Natural Connections 


\section{Creating Intelligent Robotics}

\section{Key Barrier: TRUST}

\section{Ability to predict behavior}

Explainable $\mathrm{Al}$ is often critical

Robotics Often Rules-based

Enable with Training

Reinforcement learning

Understanding enables acceptance

Support Co-Robotics

Often simple rules for complex tasks

INL is a champion of Adaptive Intelligence

\section{Isaac Asimov's Three Laws of} Robotics (1940)

First Law: A robot may not injure a human or through inaction, allow a human to come to harm.

Second Law: A robot must obey the orders given it by human beings, unless such orders would conflict with the first law.

Third Law: A robot must protect its own existence, as long as such protection does not conflict with the first or second law.

$$
\text { CSE } 415 \text { - (c) S. Tanimoto, } 2002
$$

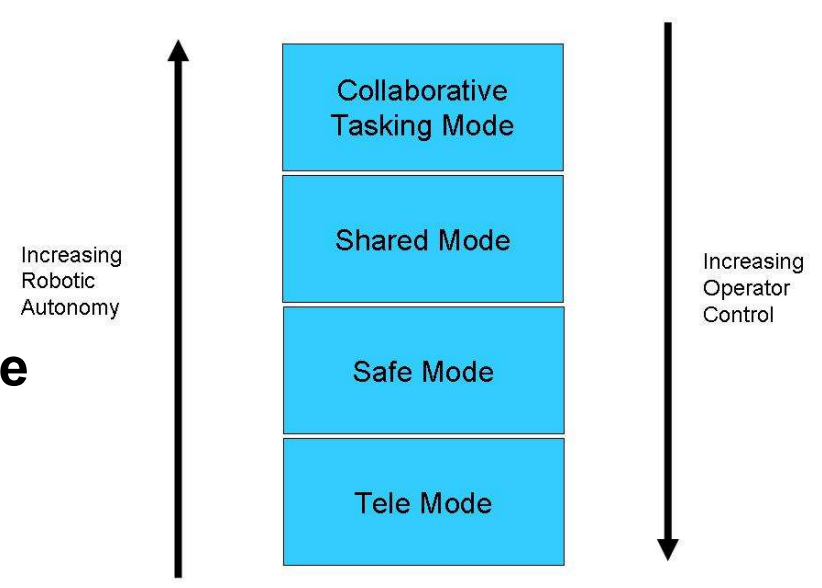

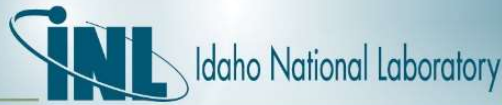




\section{Creating Intelligent Robotics}

Need ongoing research to improve robotics

Move from tool to partner

Look for Natural Connections for Human Interaction

Task-Level Execution

Focus on shared Goals / Best ability

Look for Natural Seams / Shared Cognition
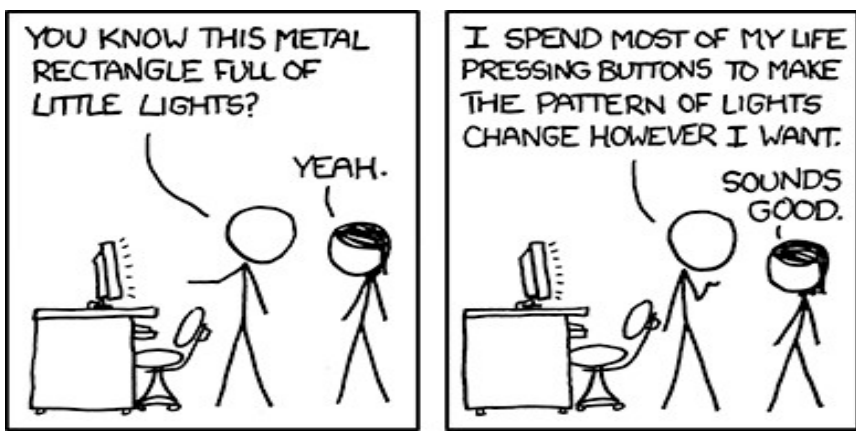

BUT TODAY, THE PATTERN OF LIGHTS IS ALL WRONG!

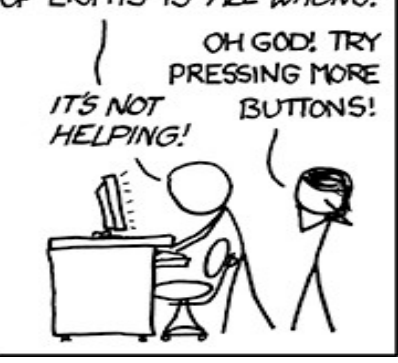

Research into more Natural Intelligence and Interaction Research in Narrative-Based Intelligence

Narratives part of Intelligence

Conclusions and Framework modelling
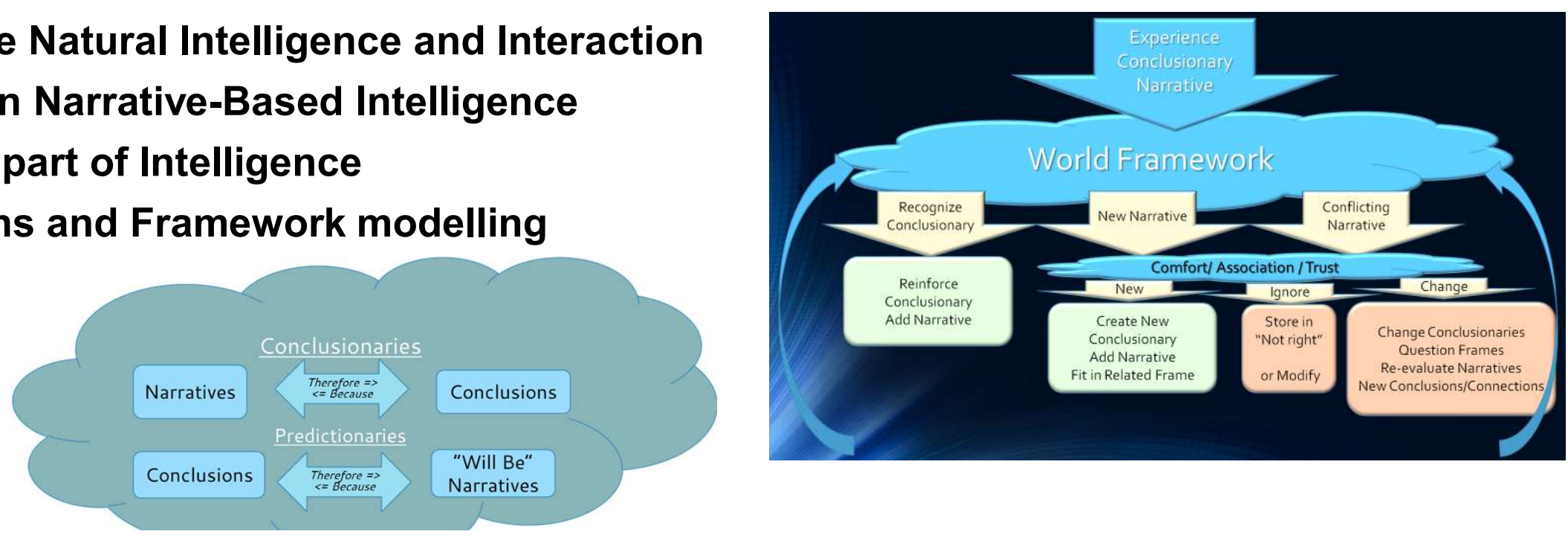


\section{Robotics Looking Ahead}

Some of INL Robotics:

Robotics Intelligence Kernel (RIK)

Counter-Mine

DOD Support

Fukushima

Tunnel Mapping

UAV work

Current/Future Research:

Hot Cell

Mobile Hot Cell

UAV work

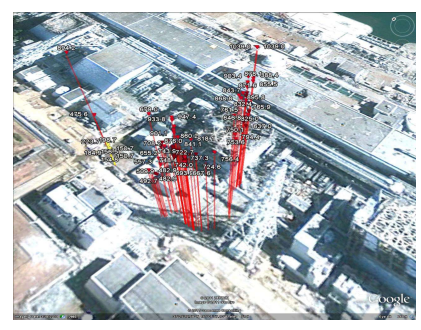

Autonomous Vehicle Impacts

Fleet Al (Caldera)

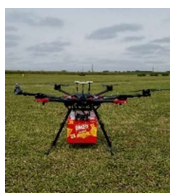

Yucca Mountain

Remote handling

Welding

Recovery

NHS Support
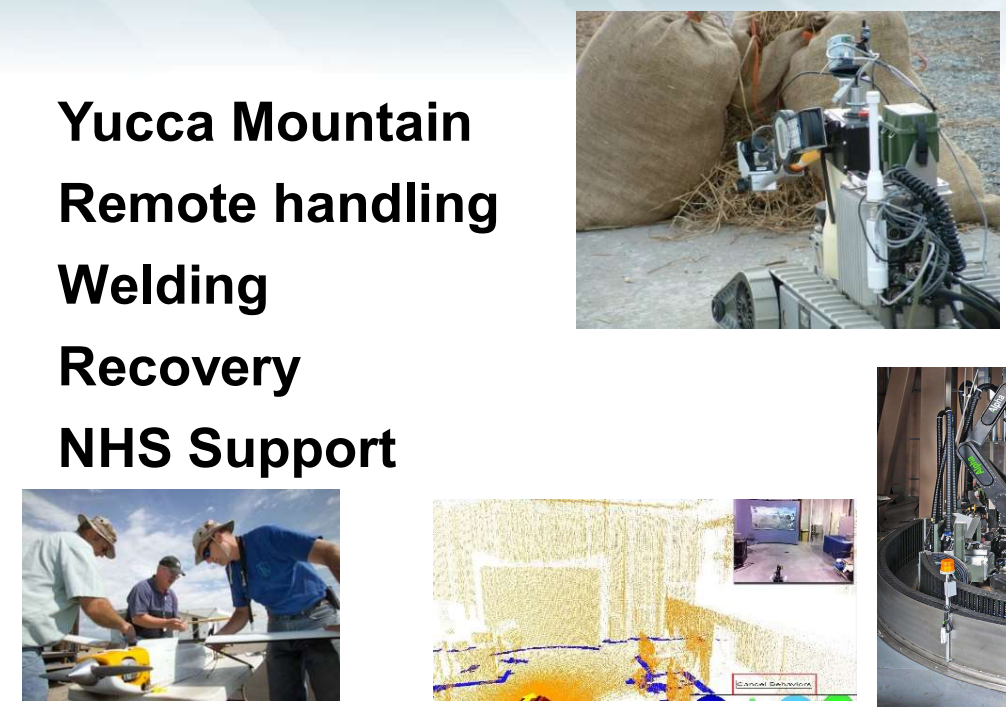

Intelligence Development

Improved partnering Enabling New Abilities

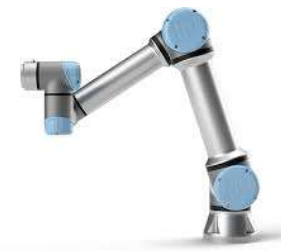

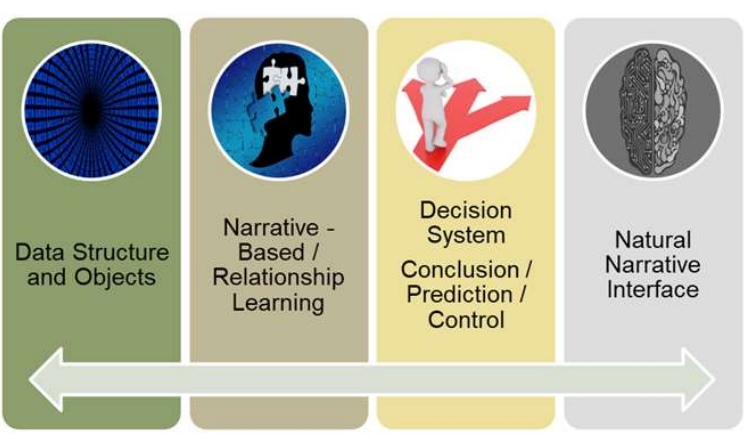


IIIIdaho National Laboratory

\section{Questions?}

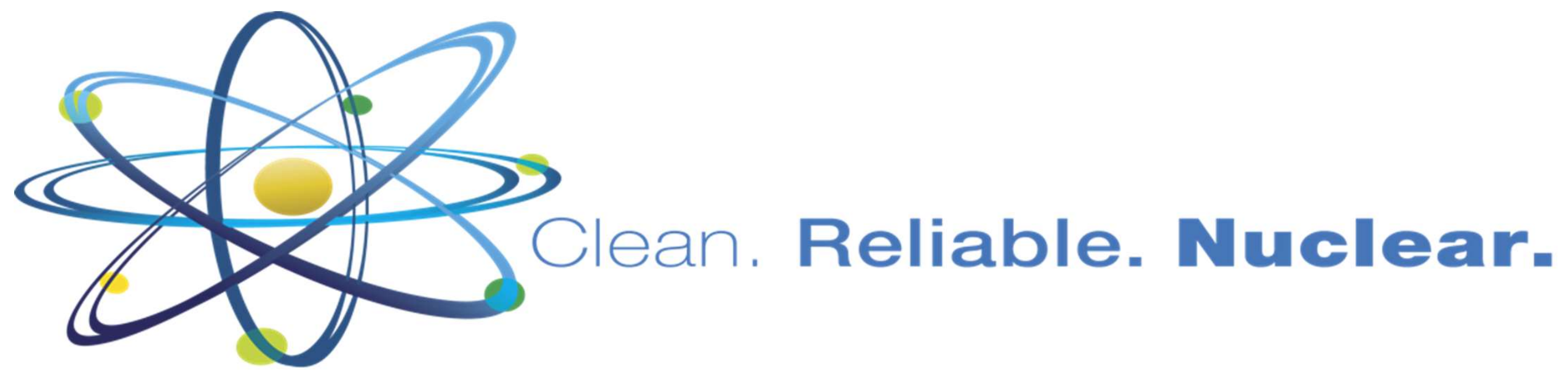




\title{
Machine Learning \& Artificial Intelligence
} Symposium

April 17, 2020

\author{
Katya Le Blanc
}

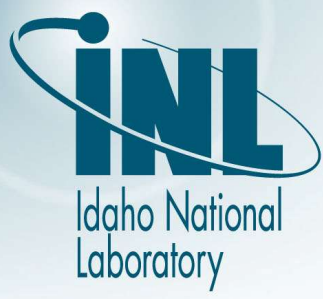




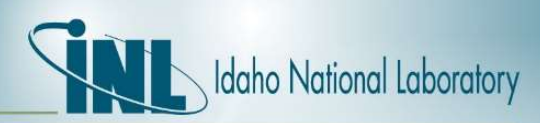

\section{Topic Introduction}

- Automation as $\mathrm{Al}$, or $\mathrm{Al}$ and Automation, or $\mathrm{Al}$ as Automation

- Discuss how Al can be used in automation

- Discuss how some existing automation, is in a sense, Al

- Discuss how we can enhance automation with $\mathrm{Al}$, including machine learning

- Discuss the strengths and weaknesses of $\mathrm{Al}$ in the context of automation

- Why it is relevant to ML/AI Future

- There is great opportunity in using $\mathrm{Al}$ in automation

- There is also great peril if we implement it poorly, especially if we don't fully understand the limitations and constraints 


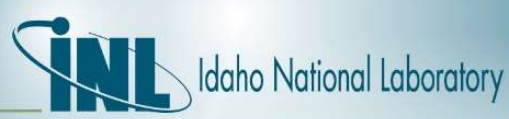

\section{Types of Al}

- Expert Systems

- Draws from human expertise to automate a task

- Typically replicates how a human would do a task

- Can help us automate tasks that humans currently do

- Machine Learning

- Perceptual Classification

- Neither approach does what humans do well, which is to develop abstract representations that we can use to generalize 


\section{Tin

\section{Expert Systems}

- Draws on expertise from multiple human experts

- More consistent than humans performing the same task

- Can be more accurate than humans, especially when human experts can supervise and update expert system with new information

- Brittle and doesn't adapt well to unforeseen situations

- Lacks insight and ability to generalize

- Many modern control systems could be classified as Al

- Draw from experts in engineering and operations and from previous experience

- Typically understandable to humans

- Depends on how systems present info
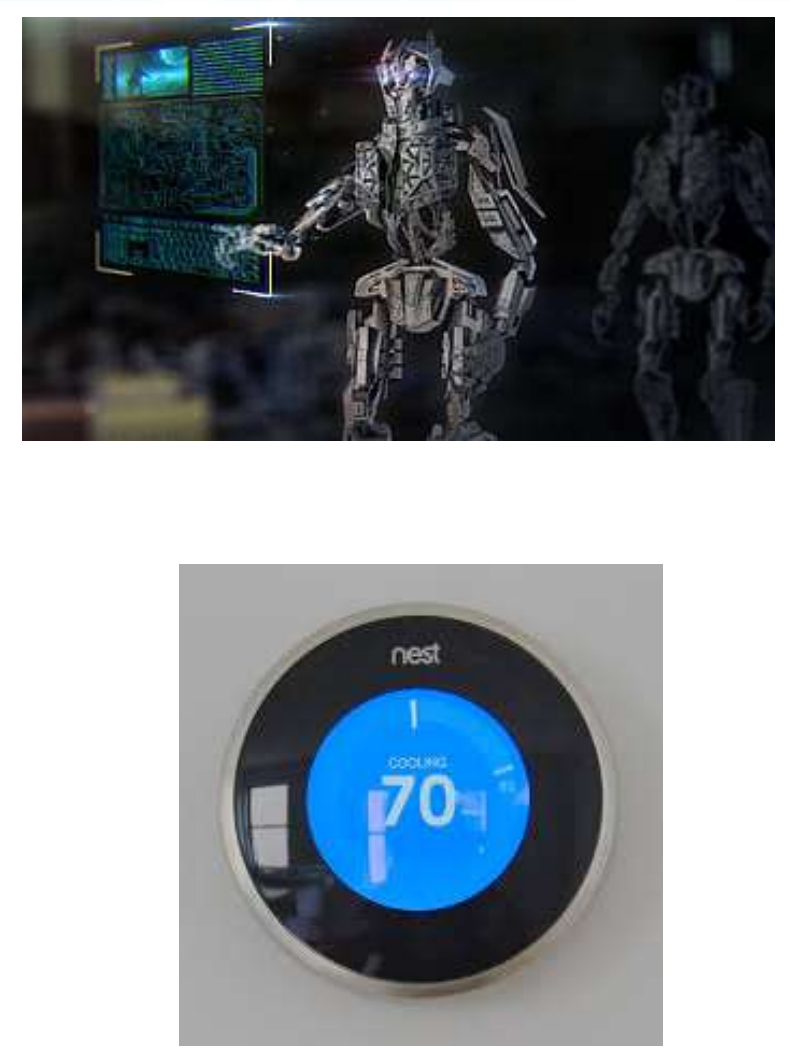


\section{Machine Learning}

- Works extremely well for well-defined classification problems

- Needs lots of data

- In contrast, humans can learn to classify with 1 example (and abstract reasoning)

- Babies learn with just a few examples

- Results depend on quality of data

- Data is not inherently objective

- Data is a human construct, we define what is collected, and what it means

- Assumptions are embedded in the data

- It does exactly what we tell it to do.....which can be a problem

- Typically opaque to humans

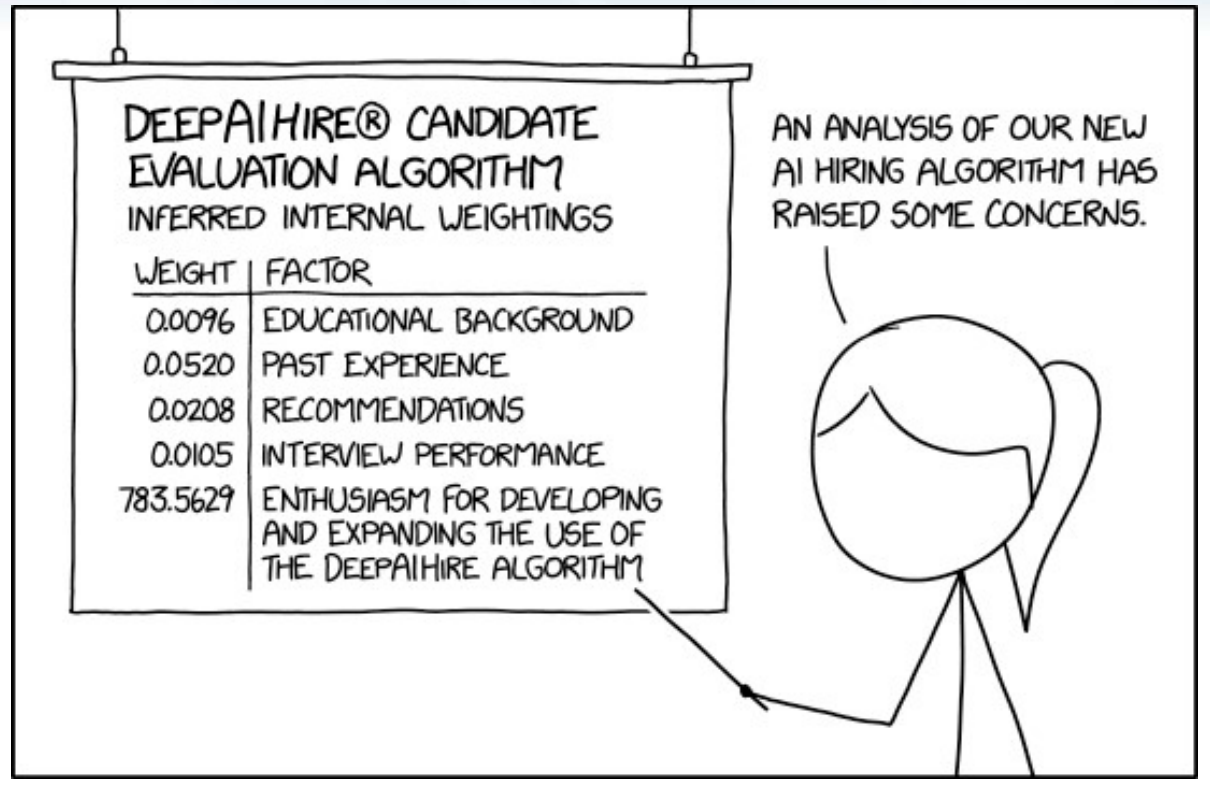

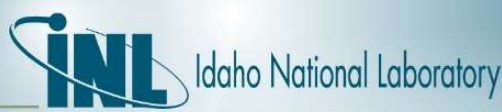




\section{Current work and future work}

- Developing expert systems to automate nuclear power plant operations (Light Water Reactor Sustainability (LWRS))

- Drawing on documentation of how humans solve problems

- Procedures

- SMES

- Operators and engineers

- Alarms and event logs

- Other data sources

- Data structure challenges

- Can we use ML to classify valid versus nuisance alarms

- Can we use ML to parse procedure text?

- Using ML and image processing for gesture recognition in

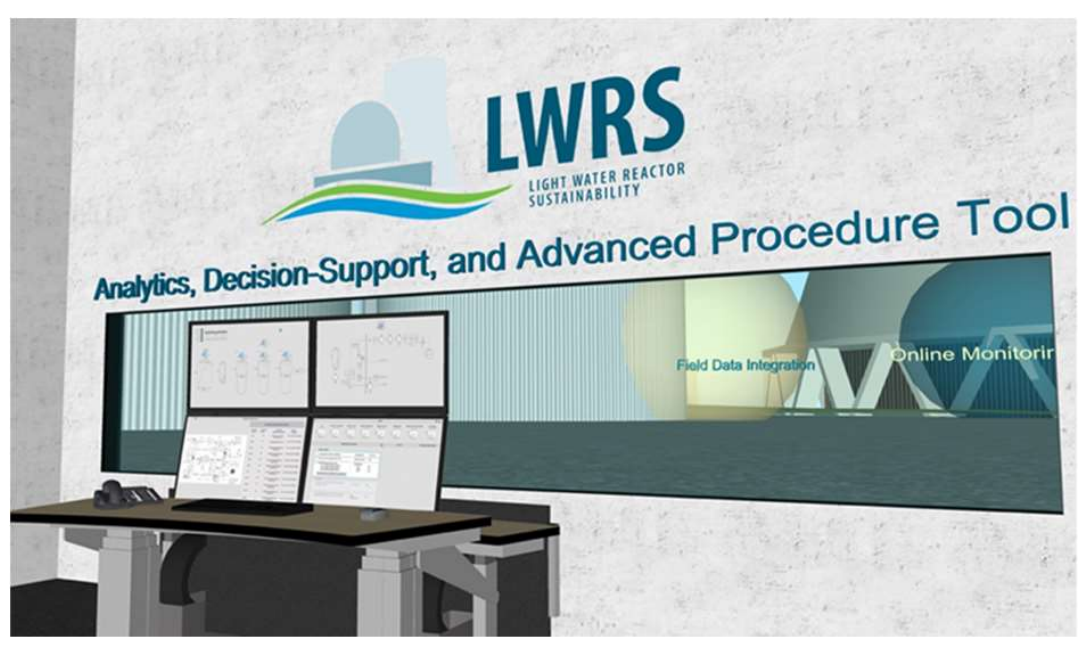
AR application for NPP field workers (Technology Commercialization Fund (TCF) Proposal with Aguiar, Yoon,\& Oxstrand)

- If we are building a system from scratch, what data should we collect and how should we structure it for maximum usefulness in some of these applications (NuScale and JUMP) 
IIIIdaho National Laboratory

\section{Questions?}

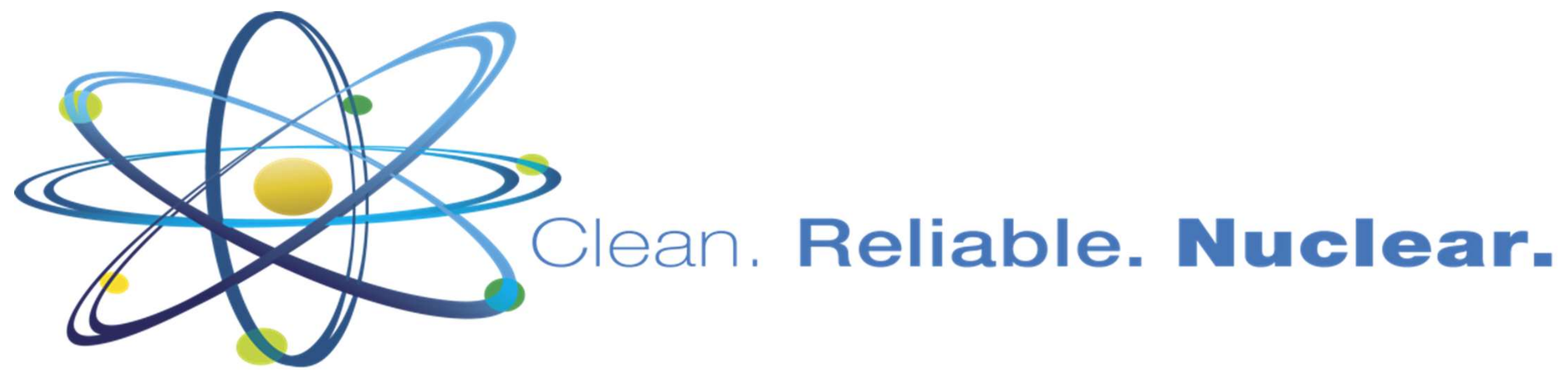




\section{Vivek Agarwal}

Group: Controls and Data Science Department within the Nuclear Safety and Regulatory Research Division

Education: B.E. degree in electrical engineering from the University of Madras, India, M.S. in electrical engineering from The University of Tennessee, Knoxville, and Ph.D. in nuclear engineering from Purdue University.

\section{Presentation Overview}

Transition from Preventive to Predictive Maintenance Strategy

- The presentation will present challenges current light water reactors are facing. How the research performed by INL in collaboration with nuclear plant owners, is providing a science-based approach to enable plant's transition from traditional labor-intensive, time- consuming preventive maintenance practice to predictive maintenance strategy. 


\title{
Machine Learning \& Artificial Intelligence Symposium April 17, 2020
}

\author{
Vivek Agarwal, PhD \\ Instrumentation, Controls, and Data Science Department (C220) \\ Transition from Preventive to Predictive Maintenance Strategy
}




\section{Diversity of Data}

- To support operation and maintenance of a nuclear power plant

- Data are collected at different spatial and temporal resolutions using different measurement techniques

- Collected data are in different format and are stored in different systems.

- Majority of the data (if not all) are collected manually.

Maintenance Strategy
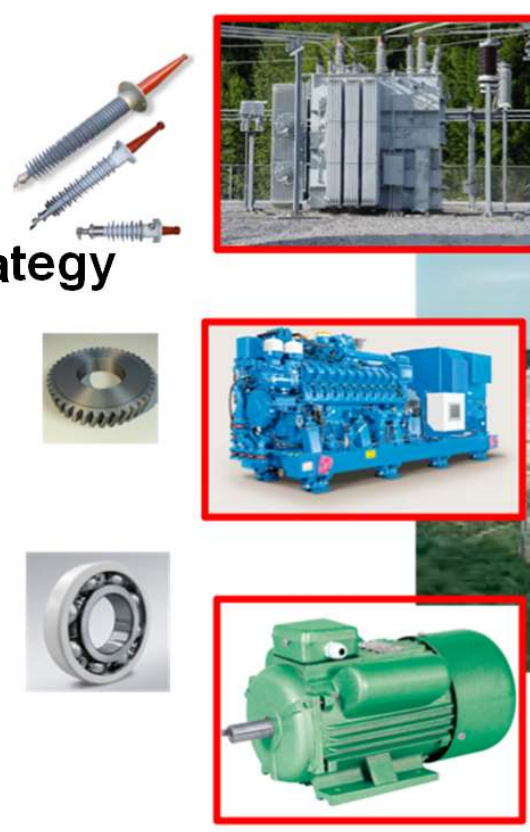
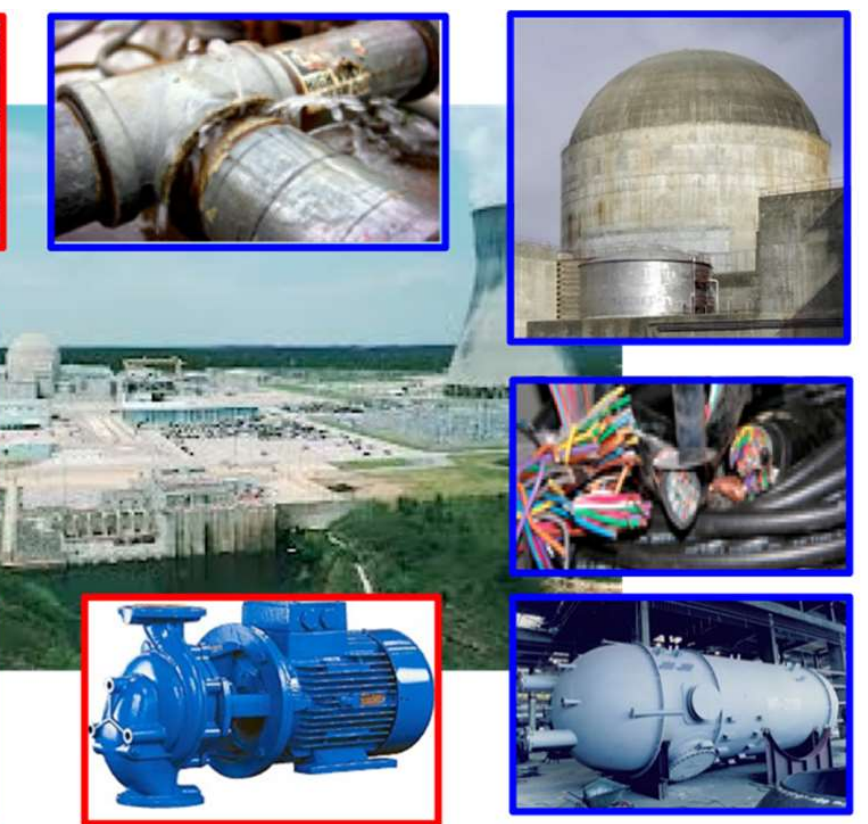

Active SSCs

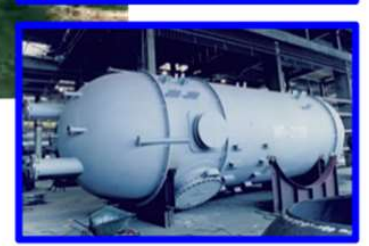

Passive SSCs
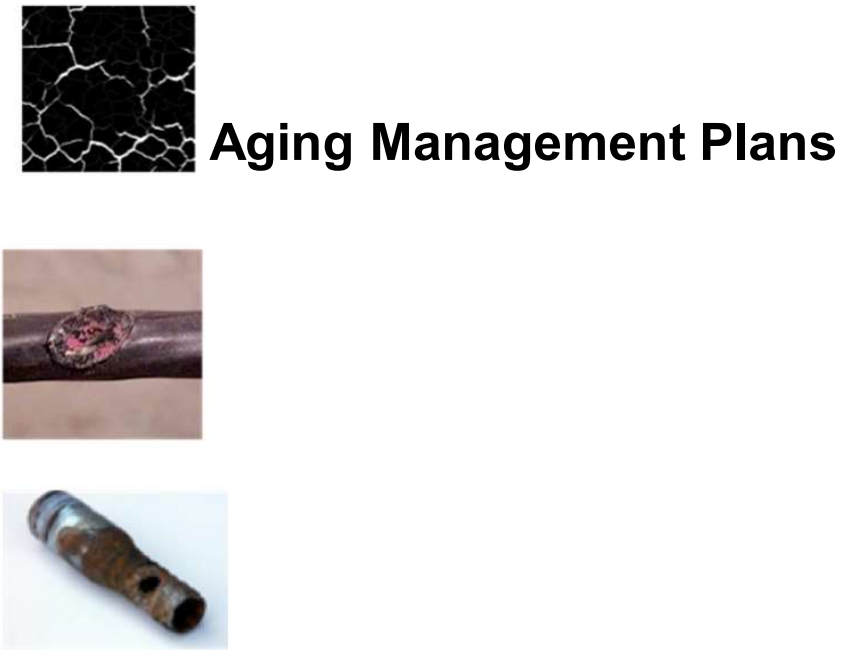


\section{Transition to Preventive to Predictive Maintenance Strategy}

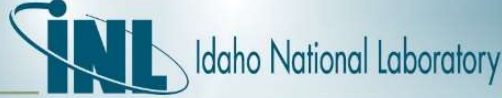

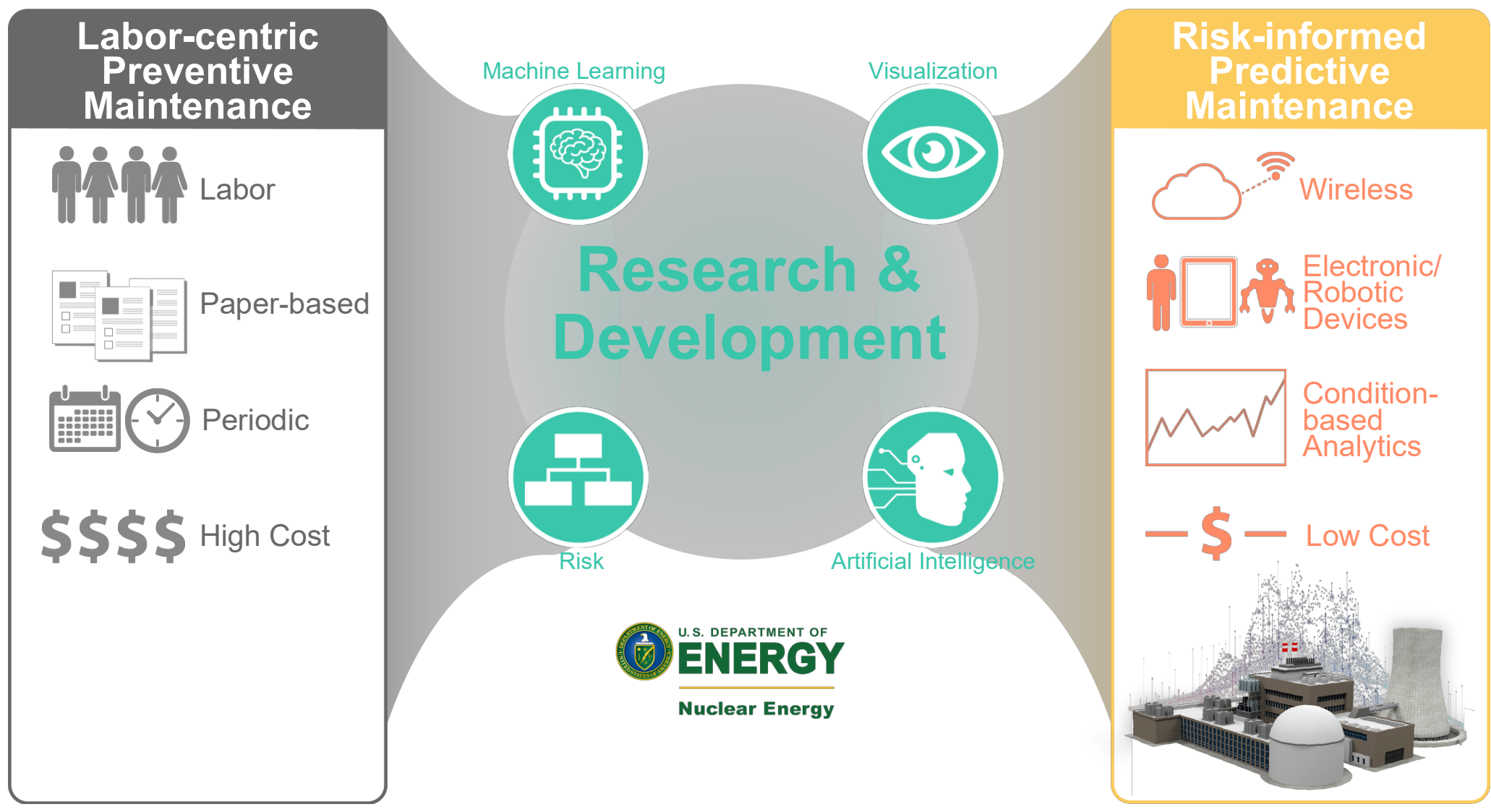

V. Agarwal et al., "Deployable Predictive Maintenance Strategy based on Models Developed to Monitor Circulating Water System at the Salem Nuclear Power Plant," INL/LTD19-55637, September 2019. 


\section{Transition from Preventive to Predictive Maintenance Strategy}
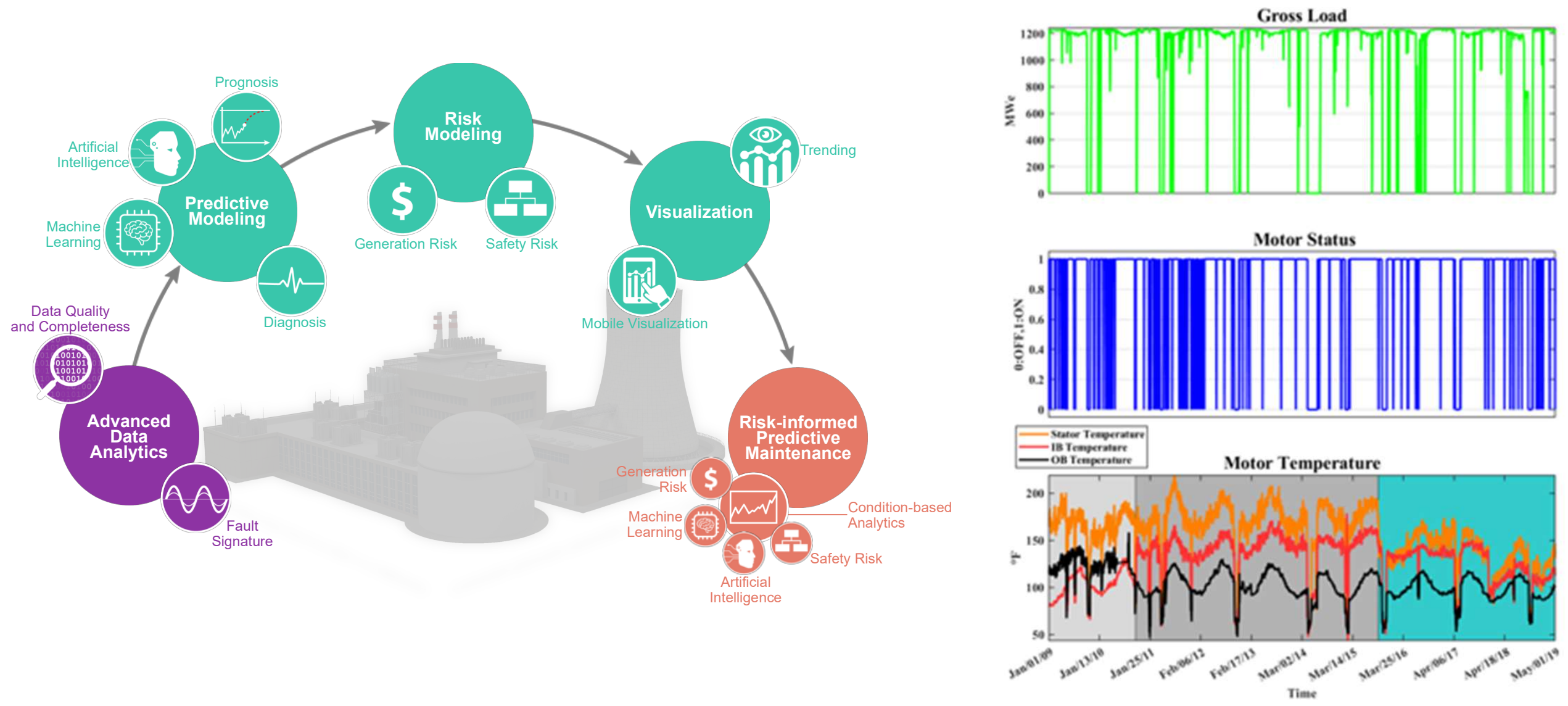


\section{Path Forward}

Scalability Analysis

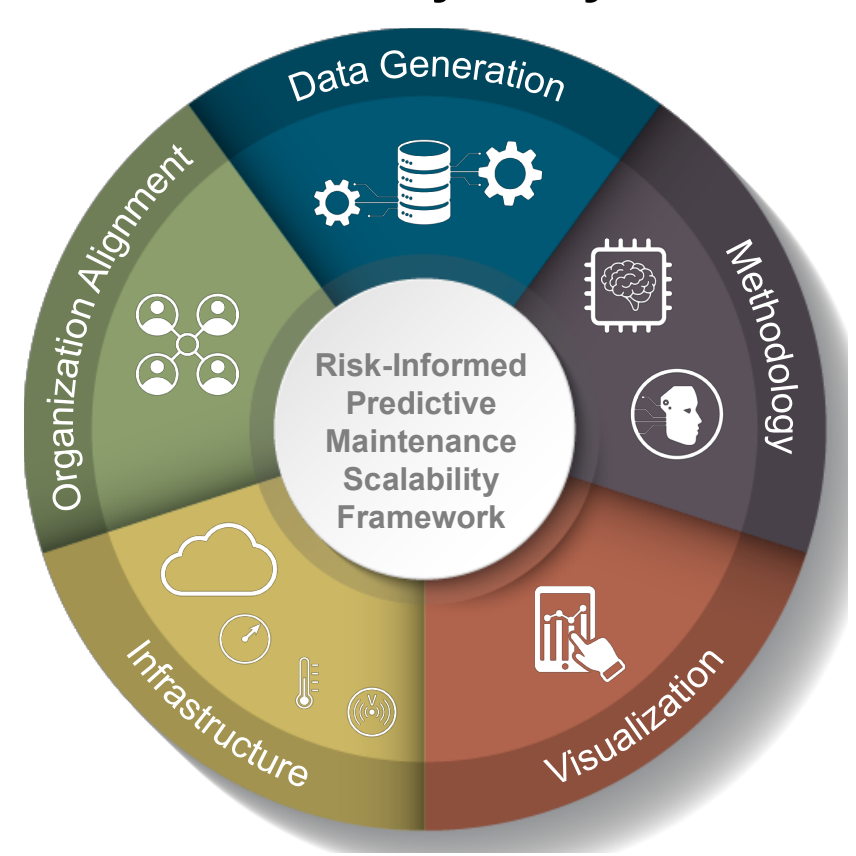

Scalability of developed approach across

- Same plant asset across the fleet and

- Different plant assets at the same plant site

\section{Multiband Heterogeneous Network ${ }^{1}$}

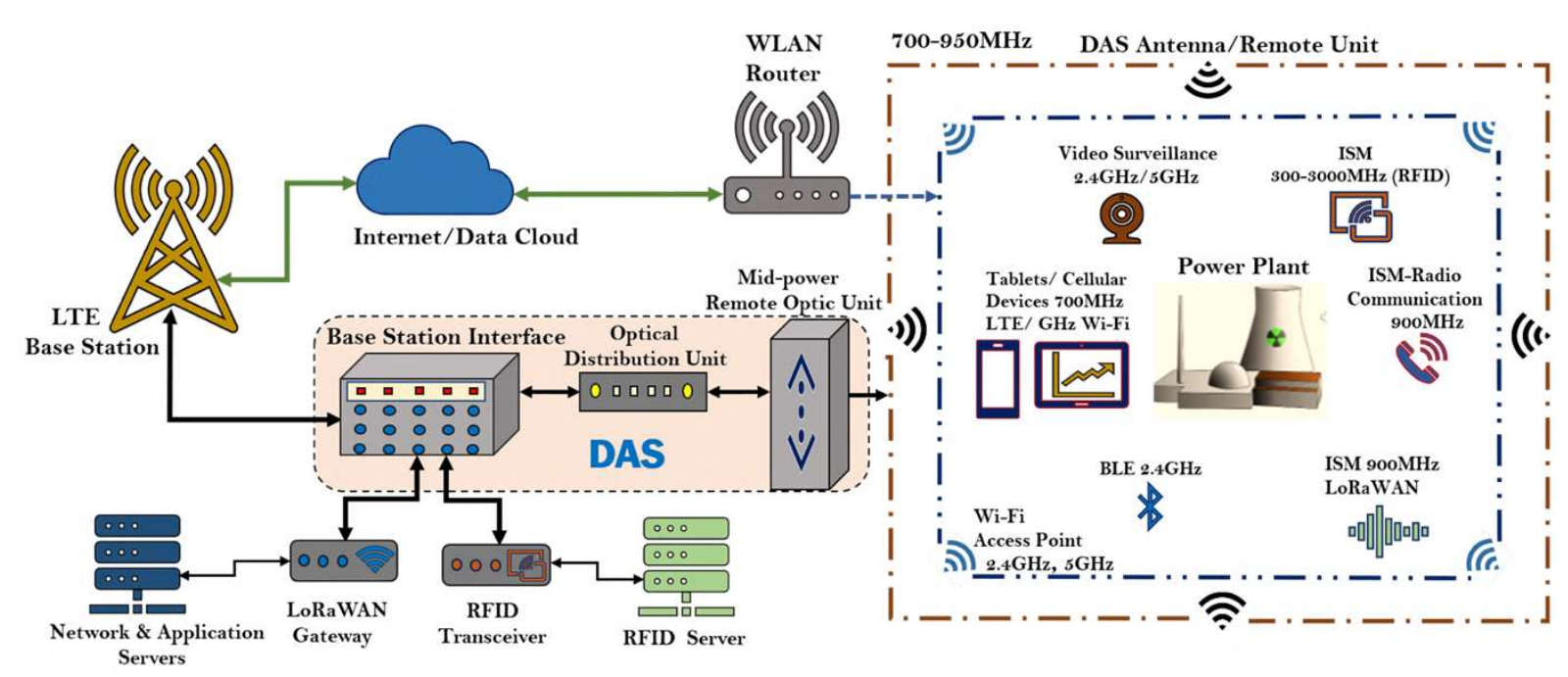

Multiband Heterogeneous Network

- low power to high power, low-frequency to high-frequency, and short-range to long-range communication regimes

${ }^{1}$ Koushik, M., and V. Agarwal, "A Multi-Band Heterogeneous Wireless Network Architecture for Industrial Automation: A Techno-Economic Analysis," INL/EXT-19-55830, September 2019. 


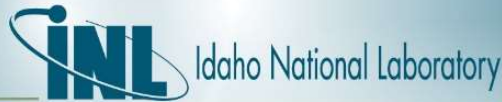

\section{End Vision}
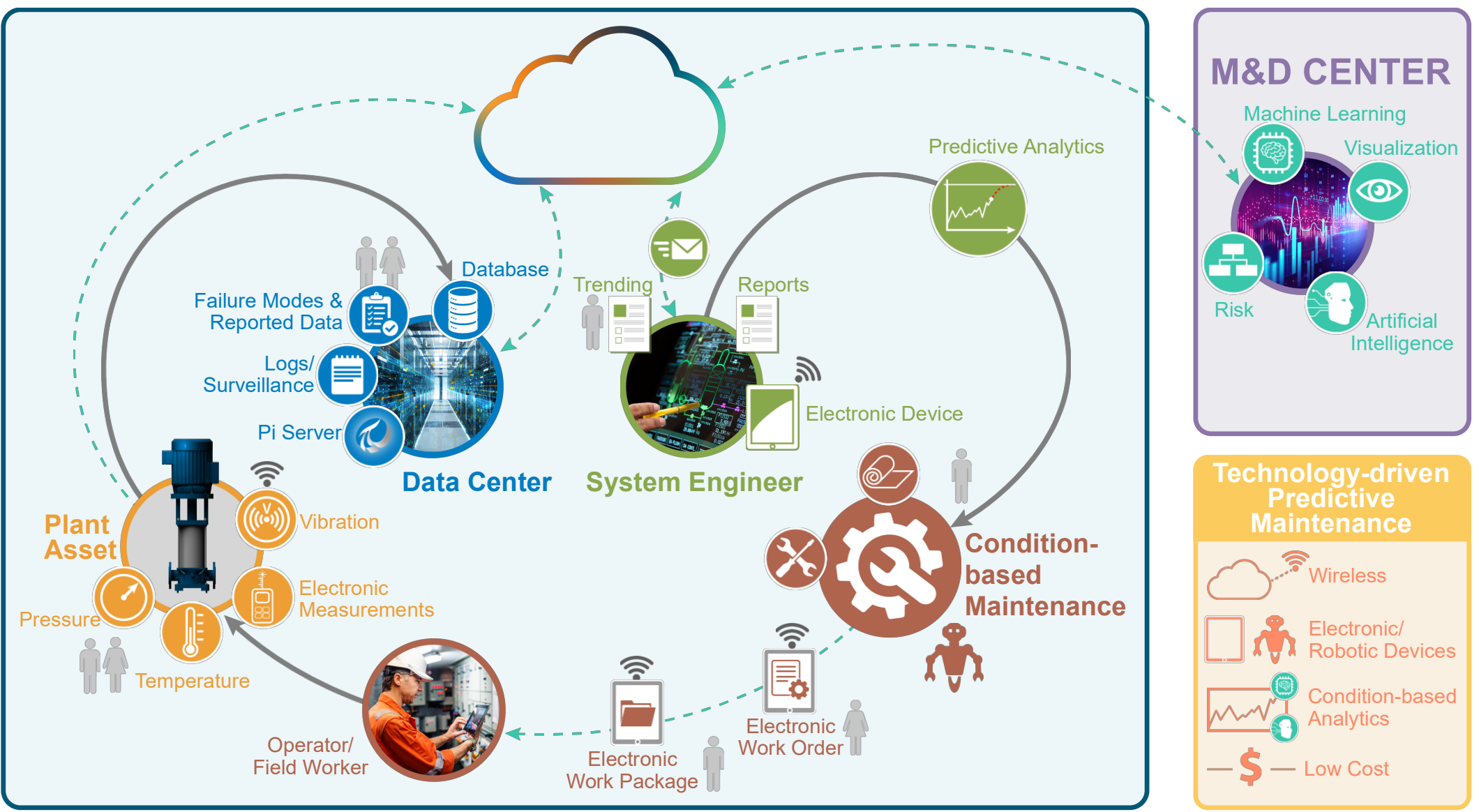


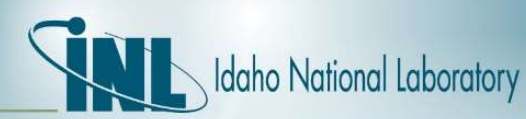

\section{Acknowledgments}

Idaho National Laboratory

- James A. Smith

- Koushik A. Manjunatha

- Vaibhav Yadav

PKMJ Technical Services

- Mathew Mackay

- Francis Lukaczyk

- Michael Archer

- Nicholas Goss

Public Service Enterprise Group, Nuclear LLC

- Palas Harry 
IIIIdaho National Laboratory

\section{Questions?}

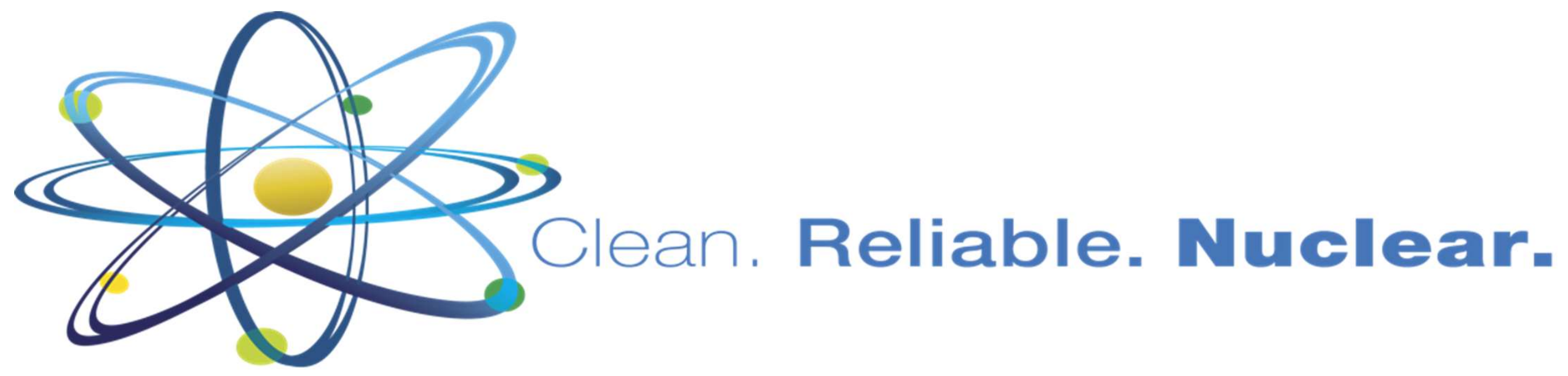




\section{Ahmad Al Rashdan}

Group: Instrumentation. Controls and Data Science Factors and Reliability

Education: Ph.D. in nuclear engineering from Texas A\&M University, a M.Sc. in information technology and automation systems from Esslingen University of Applied Science in Germany, and a B.Sc. in mechanical engineering from Jordan University of Science and Technology.

\section{Presentation Overview}

Machine Learning \& Artificial Intelligence Symposium

- Applications of Machine Learning in Automating Current Nuclear Operations and Work Processes

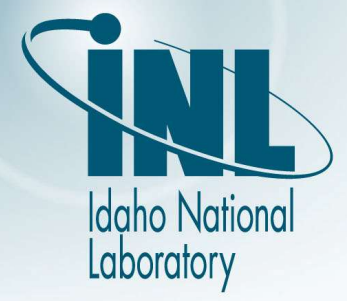


Applications of Machine Learning in Automating Current Nuclear Operations and Work Processes

April 17, 2020

Ahmad Al Rashdan, Ph.D. Instrumentation, Controls, and Data Science

Machine Learning \& Artificial Intelligence Symposium 


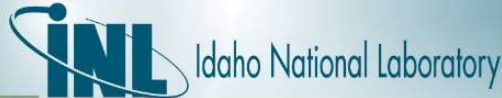

\section{Motivation}
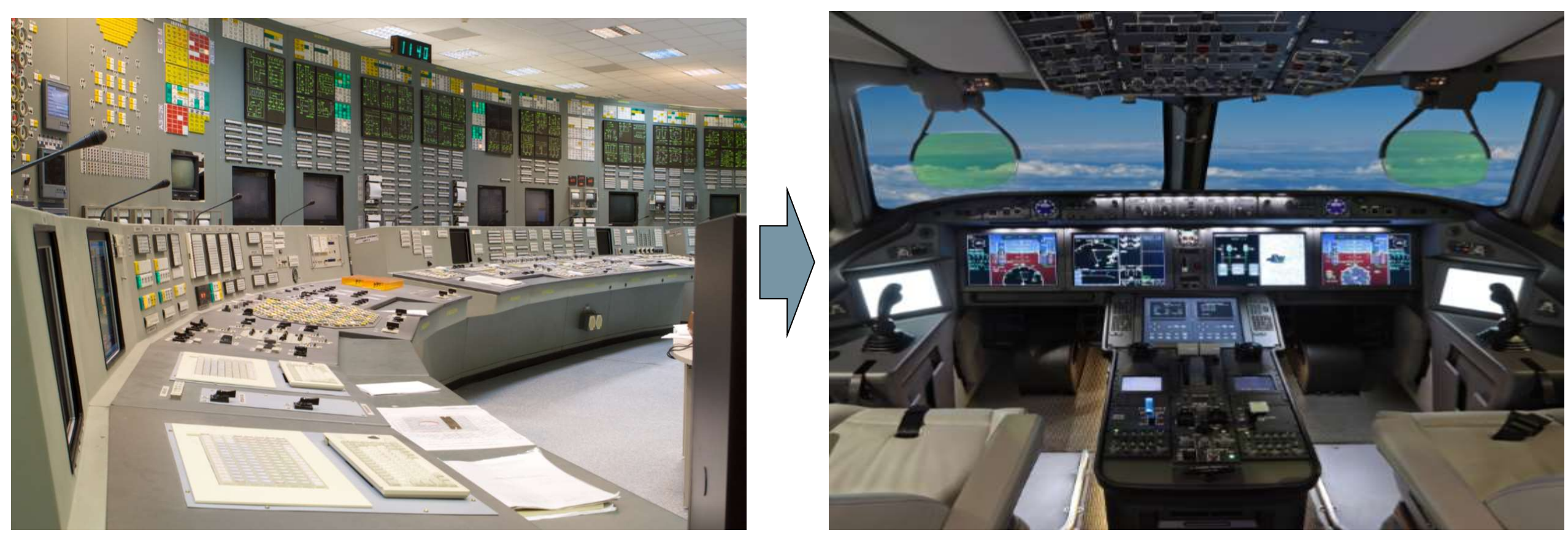

LWRS

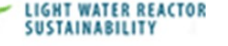




\section{Machine Learning in a Nuclear Power Plant}

Automate human activities (of visual, physical, analytical nature):

- Visual

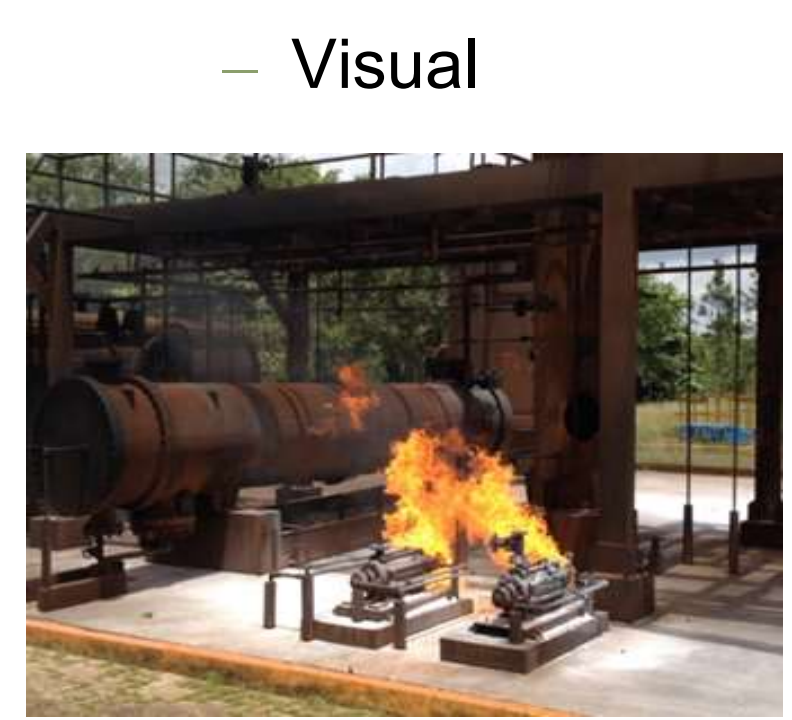

Physical

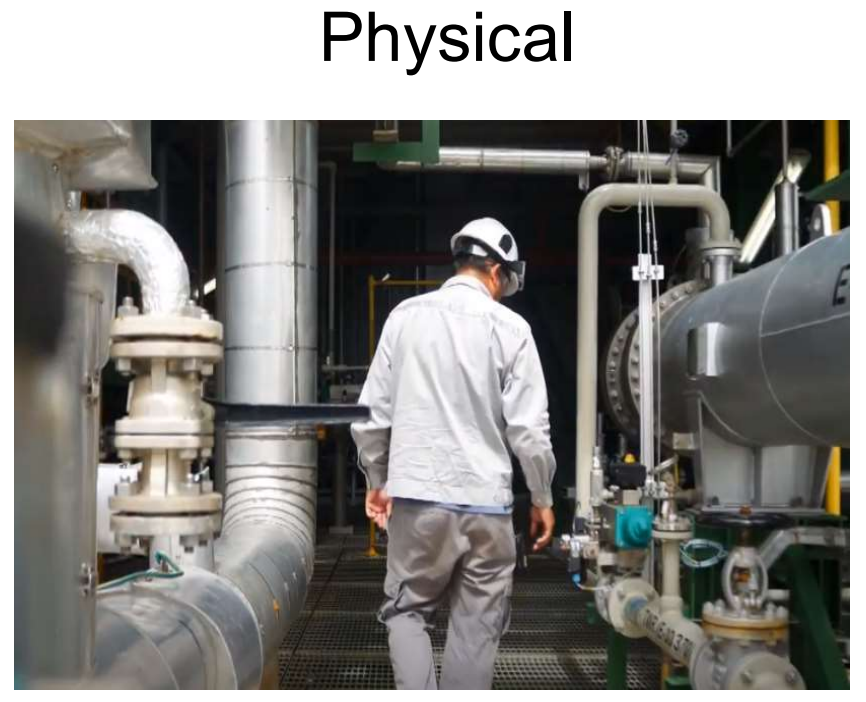

Analytical

How? perform work autonomously, faster, more frequently, more accurately, or perform tasks that a human can't perform.

\section{Tint


Types of Applications

Collection
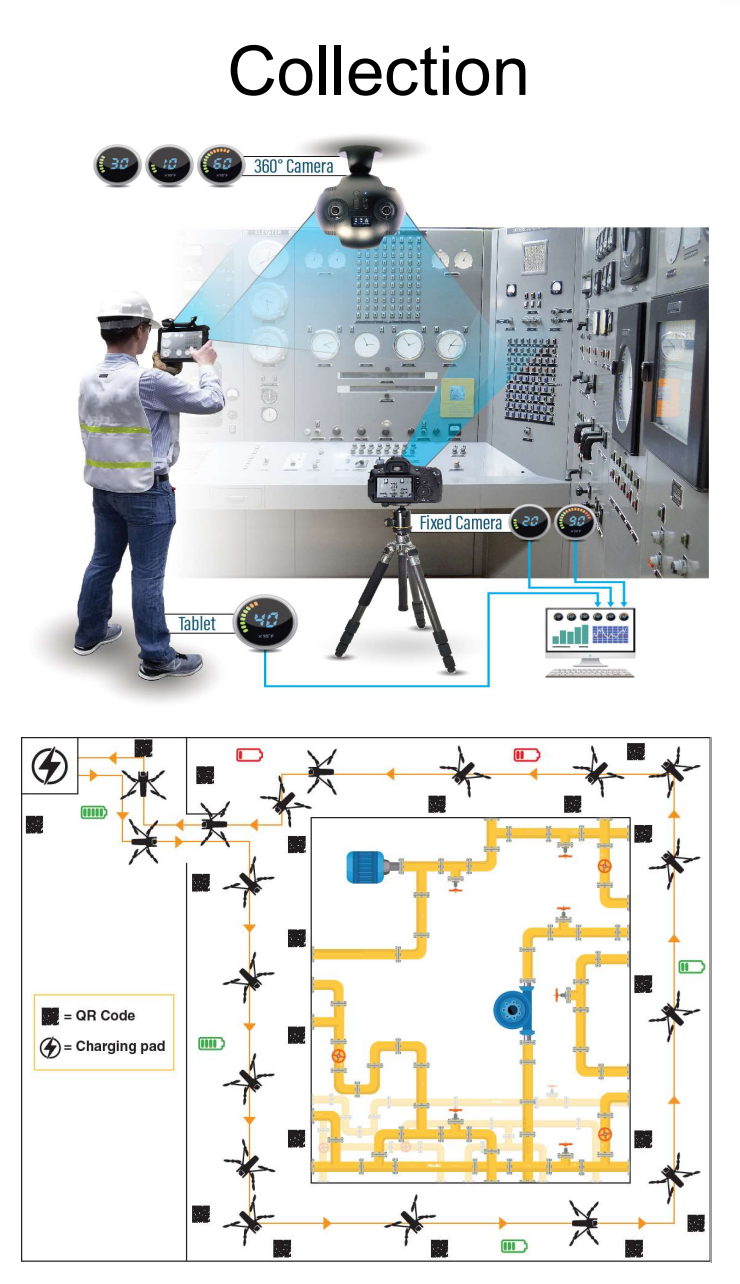

Analysis
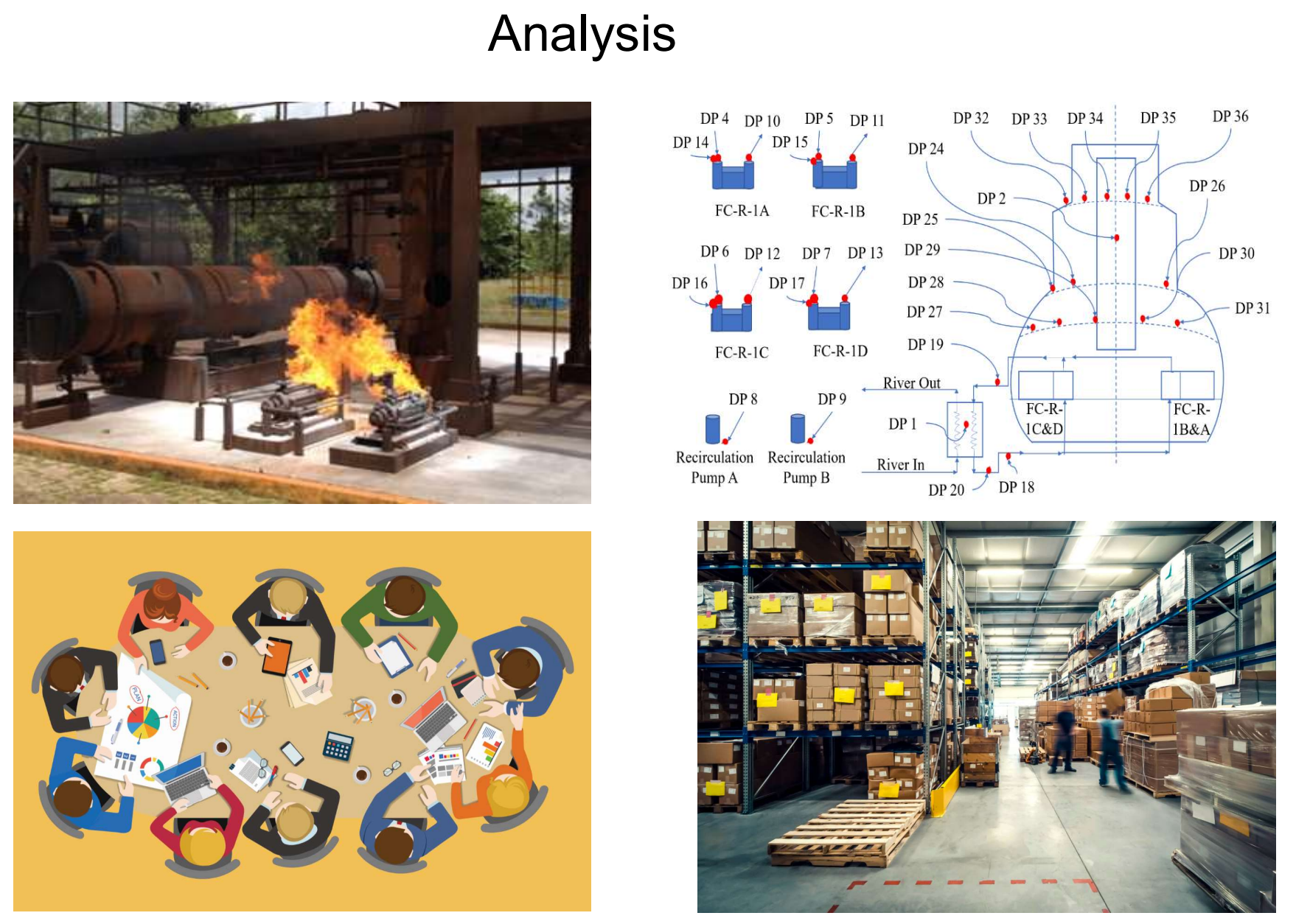

\section{Idaho National Laboratory}




\section{How does this advance ML/Al as a science?}

\section{PII}

Applied perspective on methods performance
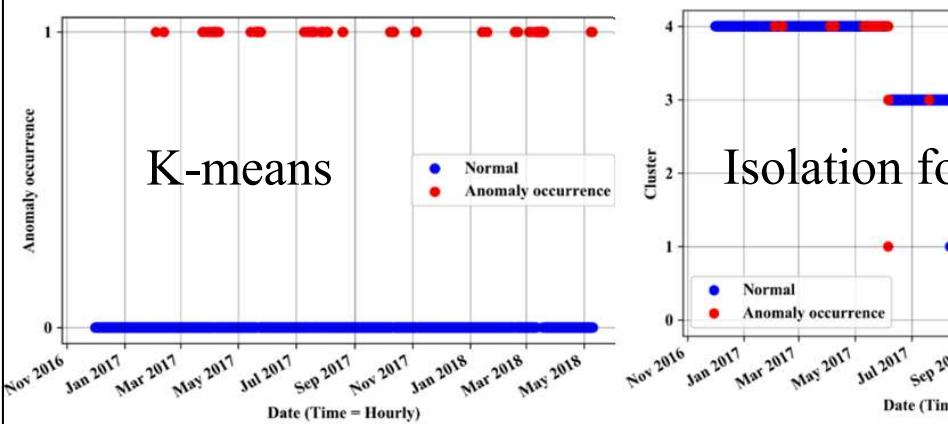

Date (Time = Hourly)

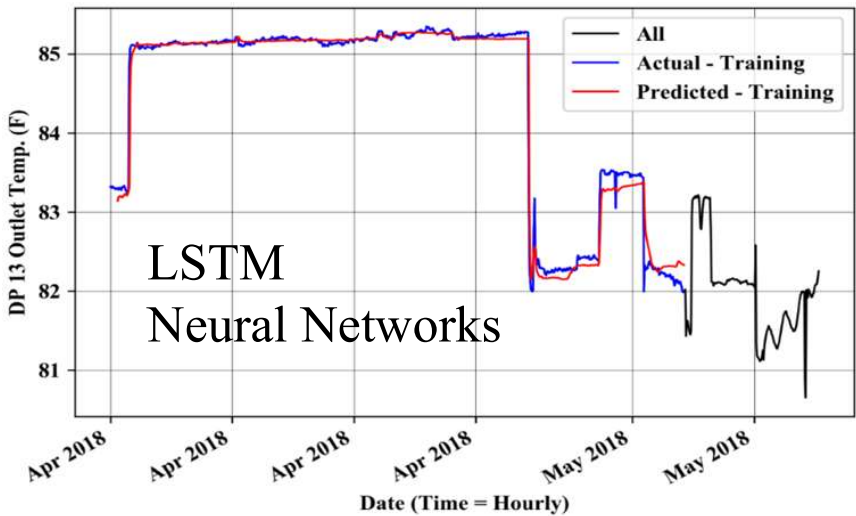

The balance between data and "physics" models
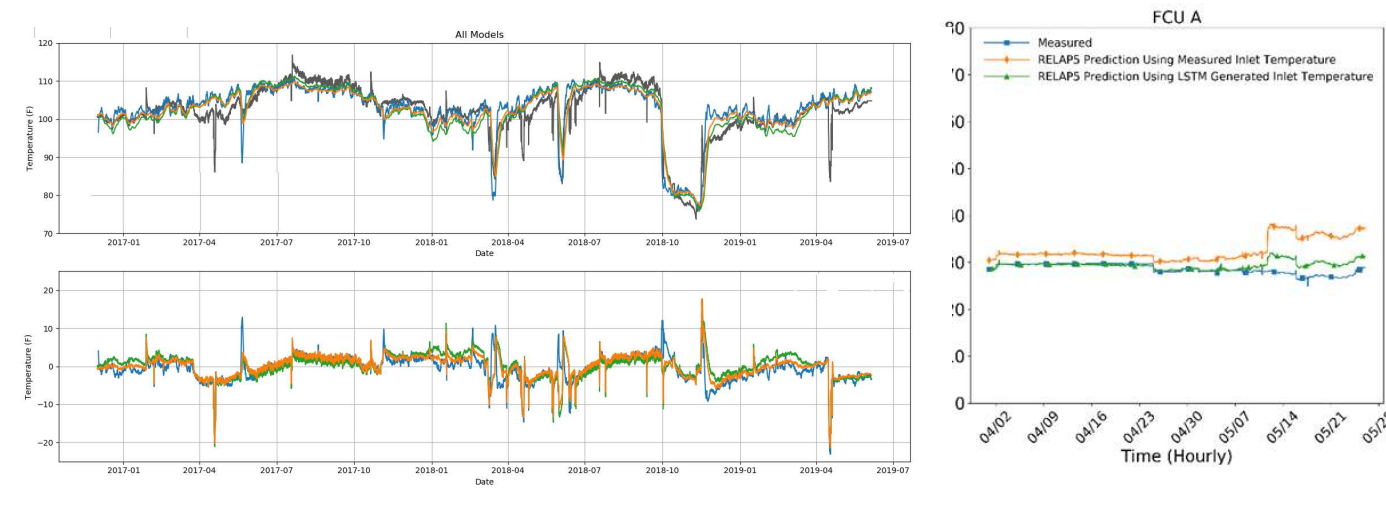

Gaps identification (looking ahead)

- Data (e.g. benchmarking)

- Methods (e.g. systematic approach)

- Verification \& Validation(e.g. overfitting)

- Deployment (e.g. computational requirements)

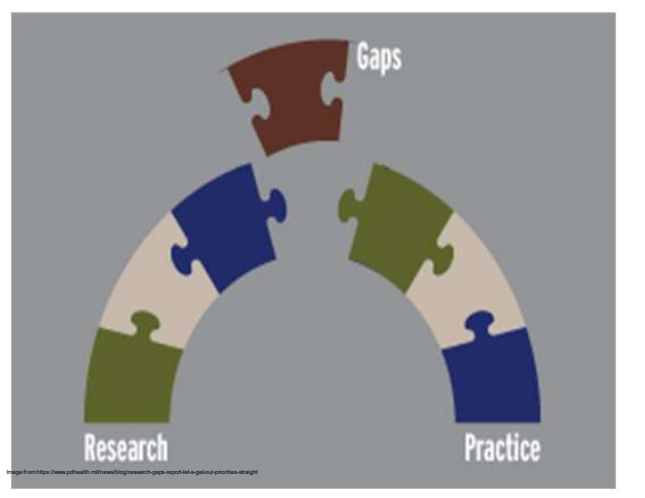


IIIIdaho National Laboratory

\section{Questions?}

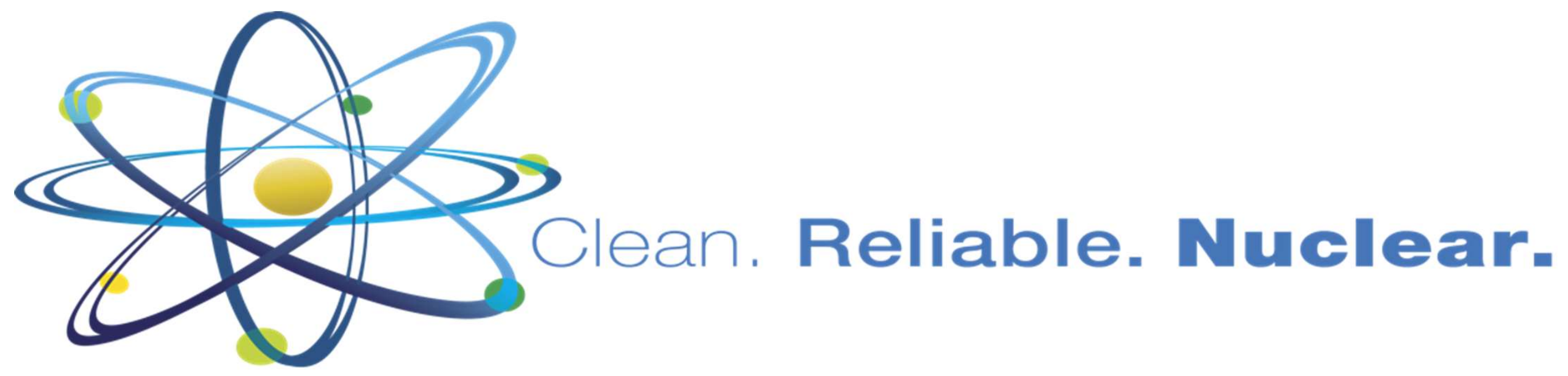




\section{Cameron Krome}

Group: High performance computing

Education: Bachelor's degree in computer science with a minor in math from Idaho State University in 2018 and is starting a master's degree in data science

\section{Presentation Overview}

Building a Scientific Language Model

- General language models like BERT and roBERTa have been extremely successful when applied to a wide range of natural language processing tasks. These models were trained using everyday language taken from blog posts, Wikipedia, etc. A language model trained instead on scientific publications from arXiv.org may perform better on tasks involving scientific research. 


\section{Machine Learning \& Artificial Intelligence Symposium April 17, 2020}

Cameron Krome

C520 - HPC Data Analytics

Idaho National Building a Scientific Language Model 


\section{Topic Introduction}

- A vast amount of data is freeform text

- Natural language processing (NLP) is a heavily focused area in ML/Al research

- The state-of-the-art methods for working with text involve general language models

- ELMo

- ULMFiT

- BERT

- rOBERTa

- Existing models are built using everyday language sources

- Blog posts

- Movie reviews

- Wikipedia

- Hypothesis:

- If we generate a language model using scientific research papers, it may perform better for tasks involving scientific data 


\section{Why it is relevant to ML/Al Future}

- Text data is generated all the time during research

- Logbooks

- Freeform text fields in databases

- Application log files

- Software

- Etc.

- The number of tasks that require working with this generated text are numerous and growing

- Problem: NLP methods change quickly

- Modifying state-of-the-art models to fit our needs can enable the lab to keep up

- Problem: The latest models are computationally expensive

- HPC resources are available for us to use if we take the time to learn how 


\section{Topic Details and Discussion}

- Retrieved scientific publications from arXiv.org - approximately 1.6 million documents

- Extracted the text from the documents

- Getting text from PDF files can be challenging

- OCR had to be performed on many documents

- Trained roBERTa from scratch using Fairseq (PyTorch) on Sawtooth GPU nodes

- Scaling is not perfect (but better than expected)

- Final model runtime on 25 nodes: $\sim 3$ weeks

- Lessons learned

- Don't worry about some bad text

- Mixed precision is essential

- Running on multiple nodes is challenging

- Checkpoint often

- Check the status of the job regularly

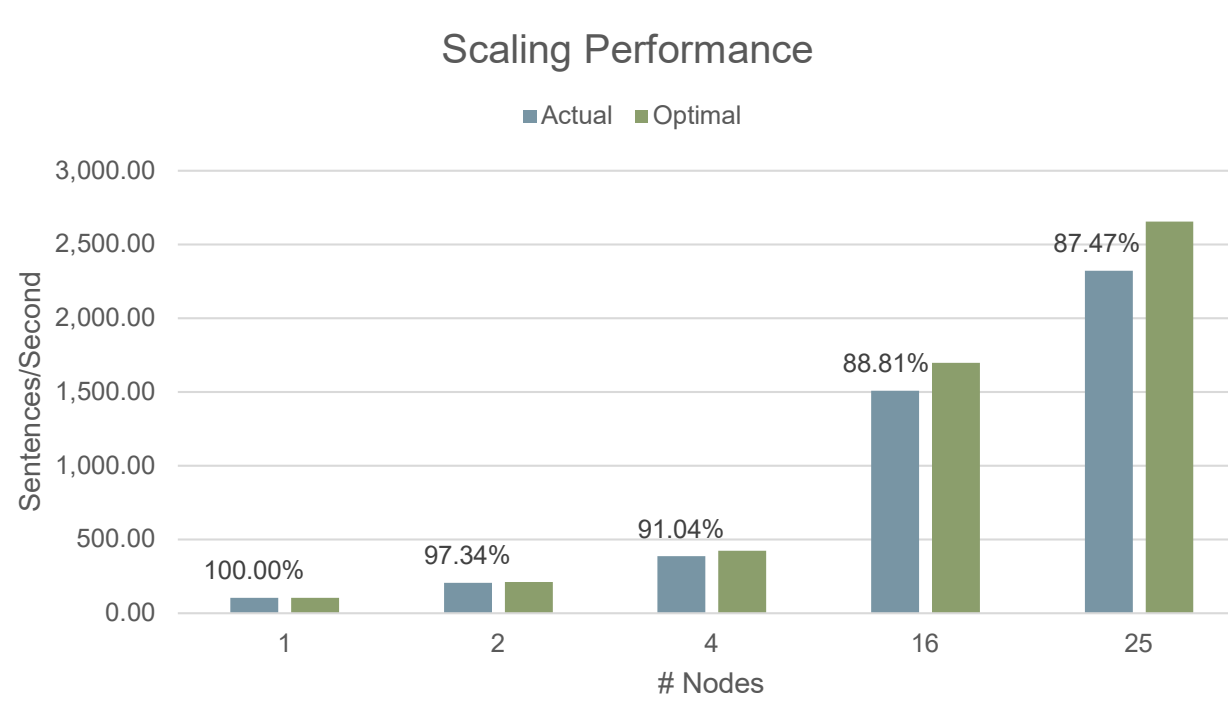




\section{Looking Ahead}

- Test the model against current benchmarks

- GLUE

- SQUAD 2.0

- CoLa

- Apply the model to INL tasks and compare against general language models

- Document classification

- Logbook analysis

- Inventory optimization

- Condition report screening

- Create other task-specific language models

- Nuclear engineering models - non-proliferation, nuclear compliance verification

- Models trained on non-word text (e.g. software, formulas, etc.)

- Explore other cutting-edge models/techniques

- Compare the performance and scalability of other libraries

- Horovod

- Tensorflow

- PyTorch 
IIIIdaho National Laboratory

\section{Questions?}

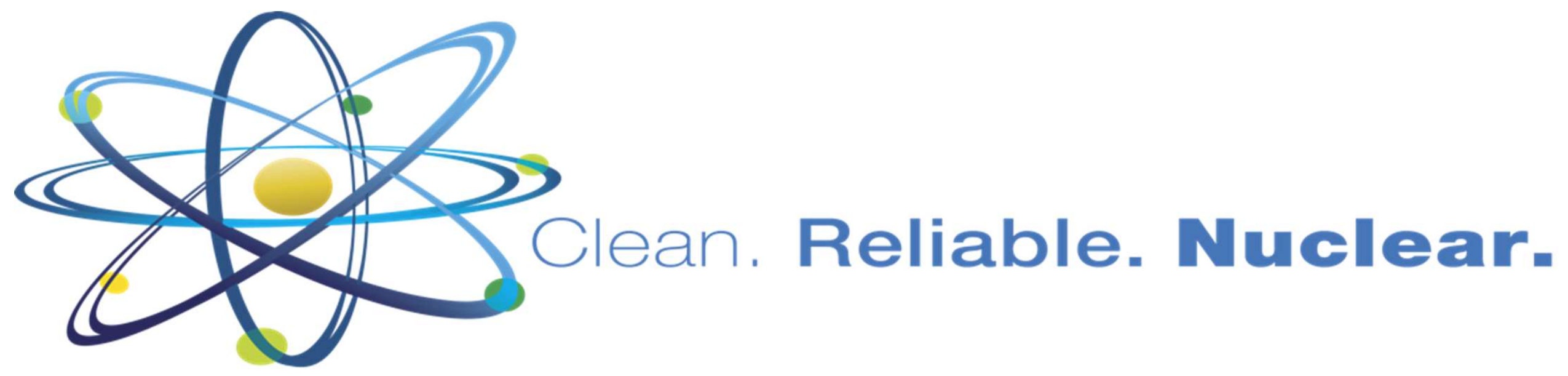




\section{Matthew Anderson}

Group: High Performance Computing C520

Education: PhD 2004, Physics, The University of Texas at

Austin

Work focused in: Reinforcement learning and deep learning

\section{Presentation Overview}

Applying Machine Learning to Code Analysis

- This talk gives a brief overview of how to apply machine learning and natural language processing to code analysis; the context of the discussion is malware analysis although the application space is much broader than just the reverse engineering of binaries. We approach the task from the perspective of machine translation with significant contributions from high performance computing and emerging hardware solutions. 


\title{
Machine Learning \& Artificial Intelligence Symposium April 17, 2020
}

\author{
Matthew Anderson \\ High Performance Computing, C520 \\ Applying Machine Learning to Code Analysis
}

Idaho National 


\section{Topic Introduction}

\section{The Challenge}

Malware and ransomware are becoming increasingly specialized and targeted. High performance computing (HPC) systems are starting to be targeted.

The challenge: rapidly identify novel malware and reduce vulnerabilities.

Examples:

- 2003 -- 2005: "Stakkato" attack against DOE, National Center for Atmospheric Research, and National Science Foundation (NSF) HPC sites

- 2014: Two NSF HPC sites were compromised by a US researcher.

- 2014-2017: "Cloud Hopper" attacks access the internal networks at Hewlett Packard Enterprise (HPE) and IBM and accessed customer systems.

- 2018: Nuclear scientists using the HPC system at the Federal Nuclear Center in Sarov Russia arrested for bitcoin mining.

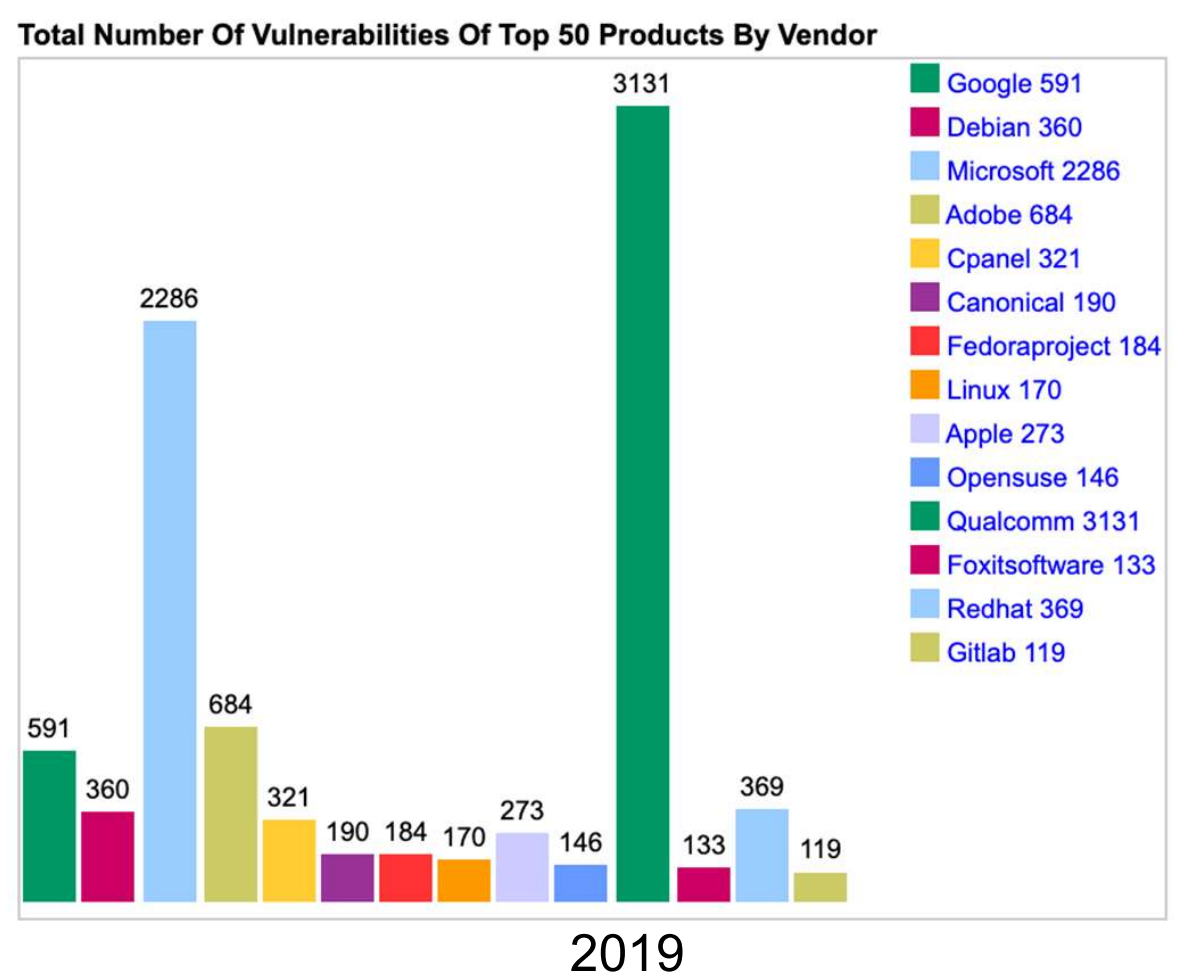




\section{Why it is relevant to ML/AI Future}

\section{The Naturalness Hypothesis}

"Software is a form of human communication; software corpora have similar statistical properties to natural language corpora; and these properties can be exploited to build better software engineering tools."

-- M. Allamanis, E. Barr, P. Devanbu, and C. Sutton (2017) arxiv.org/pdf/1709.06182.pdf

The outcome: Apply Natural Language Processing (NLP) and Machine Learning techniques to software! Some Examples:

Source code analysis

Binary analysis

\begin{tabular}{|l|c|c|c|c|}
\hline Reference & $\begin{array}{c}\text { Predicting Program } \\
\text { Bugs }\end{array}$ & $\begin{array}{c}\text { Synthesizing } \\
\text { patches and code } \\
\text { changes }\end{array}$ & $\begin{array}{c}\text { Identifying function } \\
\text { signatures }\end{array}$ & $\begin{array}{c}\text { Addressing Code } \\
\text { Obfuscation }\end{array}$ \\
\hline Dam (2018) & $\checkmark$ & & $\begin{array}{c}\text { Recovering } \\
\text { compiler used to } \\
\text { generate binary }\end{array}$ \\
\hline Chakraborty (2018) & $\checkmark$ & $\checkmark$ & $\checkmark$ & $\checkmark$ \\
\hline Ding (2019) & & & $\checkmark$ & $\checkmark$ \\
\hline Massarelli (2019) & & & & \\
\hline
\end{tabular}




\section{Topic Details and Discussion}

\section{Challenges in Binary Analysis}

1. Function names and debug symbols are stripped out from the binary

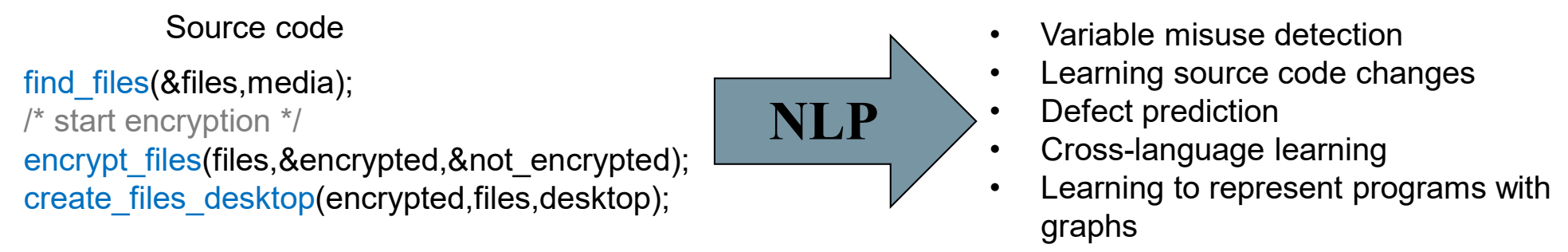

2. In real-life cases, we have to undo code obfuscation

Common Code Obfuscations: • Packing

- Adding bogus logics

- Splitting basic blocks

- Substituting instructions

- Bogus control flow graphs

- Hot patching mechanisms (e.g. Conficker)

3. Assembly functions may appear different but still share the same functional logic

Addr_1: mov eax,10

Addr 2: dec eax

Addr_3: mov [base+eax],0

Addr_4: jnz Addr_2

Addr_5: mov eax,ebx

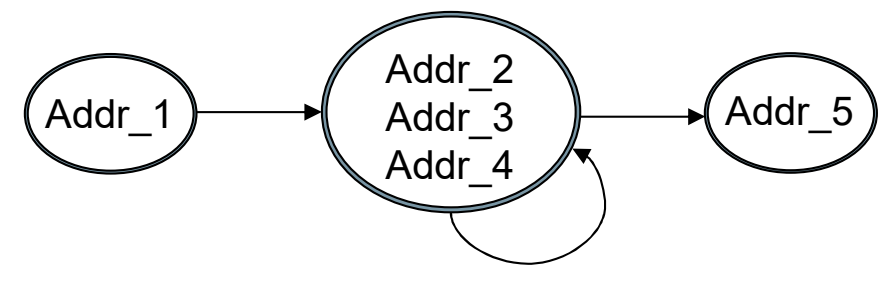




\section{Topic Details and Discussion}

\section{The Clones Ansatz:}

"Just as there is uncontrolled software reuse in source code, there exists a large number of clones in the underlying assembly code as well."

S. Ding, B. Fund, P. Charland (2019)

Binary code fingerprints: four types of assembly code similarities

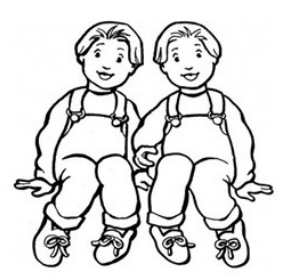

Literally Identical

$$
\begin{aligned}
& i++ \\
& i=i+1
\end{aligned}
$$

Syntactically Equivalent

Opportunities for Deep Learning:

-- Identify binary similarities

-- Assign probable function names

-- Rapid identification of novel malware

-- Identification of software vulnerabilities memcpy Same strcpy source memncpy or with/without mempcpy obfuscation

Semantically Similar 


\section{Topic Details and Discussion}

\section{Datasets:}

\section{Datasets, Tools, and Approach}

- Vulnerability dataset: Contains 3,015 assembly functions compiled with various compilers; contains variants of Heartbleed, Shellshock, Venom, Clobberin' Time, etc.

- UbuntuDataset: 87,853 ELF files disassembled using IDA Pro with $>10$ million distinct named functions

- NERO: 13,826 named functions from GNU repository with control flow graphs

- Research Malware/Ransomware: GonnaCry, Mirai

Tools:

DeepGraphLibrary asm2vec angr

\section{Approach:}

- Approach binary analysis (binary similarity, function naming) using Neural Machine Translation:

- Bidirectional recurrent neural network with Long Short-Term-Memory cells

- Incorporate the Transformer Architecture

- Augment existing datasets with Github projects (>28 million public repositories) and more malware

- Create new metrics for scoring semantic similarity in binaries akin to what is used in NLP (e.g. BERTScore T. Zhang et al. 2020). 
IIIIdaho National Laboratory

\section{Questions?}

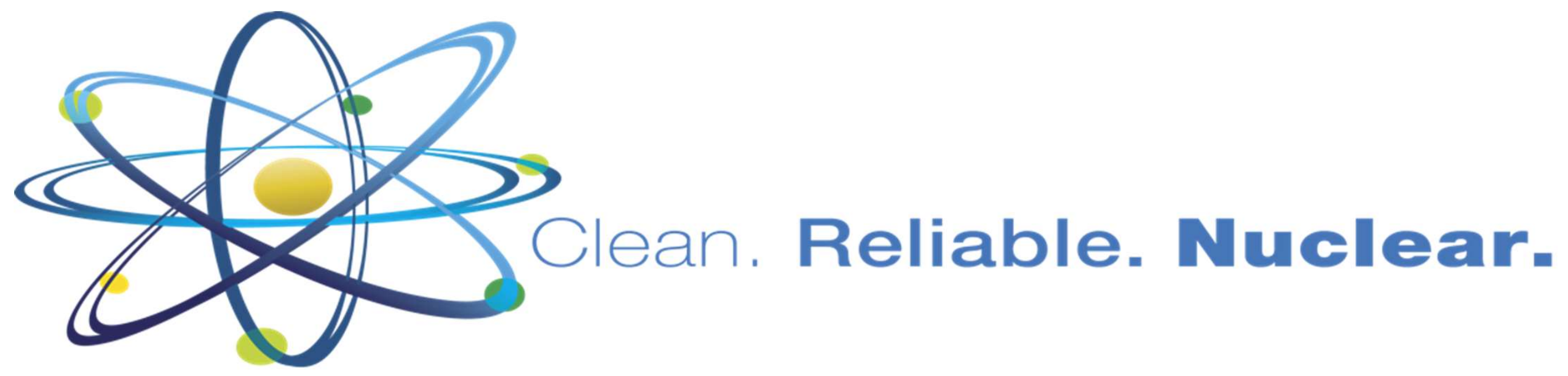

